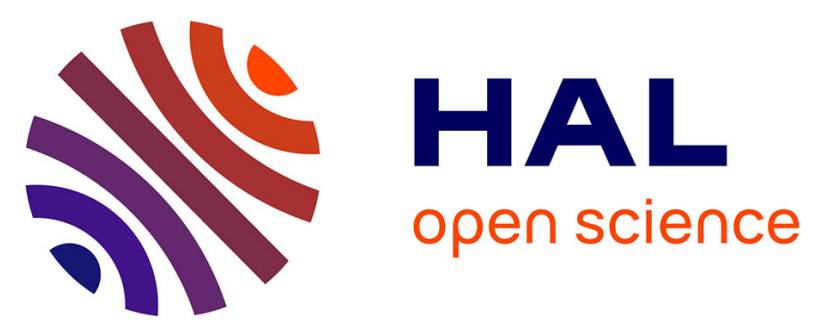

\title{
The ALMA Spectroscopic Survey in the Hubble Ultra Deep Field: Evolution of the Molecular Gas in CO-selected Galaxies
}

Manuel Aravena, Roberto Decarli, Jorge Gónzalez-López, Leindert Boogaard, Fabian Walter, Chris Carilli, Gergö Popping, Axel Weiss, Roberto J Assef, Roland Bacon, et al.

\section{To cite this version:}

Manuel Aravena, Roberto Decarli, Jorge Gónzalez-López, Leindert Boogaard, Fabian Walter, et al.. The ALMA Spectroscopic Survey in the Hubble Ultra Deep Field: Evolution of the Molecular Gas in CO-selected Galaxies. The Astrophysical Journal, 2019, 882 (2), pp.136. 10.3847/1538-4357/ab30df . hal-02325118

\section{HAL Id: hal-02325118 \\ https://hal.science/hal-02325118}

Submitted on 22 Oct 2019

HAL is a multi-disciplinary open access archive for the deposit and dissemination of scientific research documents, whether they are published or not. The documents may come from teaching and research institutions in France or abroad, or from public or private research centers.
L'archive ouverte pluridisciplinaire HAL, est destinée au dépôt et à la diffusion de documents scientifiques de niveau recherche, publiés ou non, émanant des établissements d'enseignement et de recherche français ou étrangers, des laboratoires publics ou privés. 


\title{
The ALMA Spectroscopic Survey in the Hubble Ultra Deep Field: Evolution of the Molecular Gas in CO-selected Galaxies
}

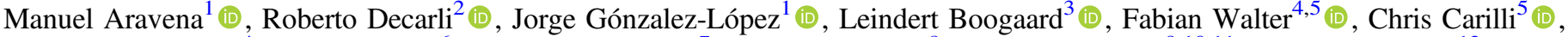 \\ Gergö Popping ${ }^{4}$ (1), Axel Weiss ${ }^{6}$ (10), Roberto J. Assef ${ }^{7}$ (1), Roland Bacon $^{8}$, Franz Erik Bauer ${ }^{9,10,11}$ (10), Frank Bertoldi ${ }^{12}$ (1), \\ Richard Bouwens $^{3}$ (10), Thierry Contini ${ }^{13}$ (1), Paulo C. Cortes ${ }^{14,15}$ (1) , Pierre Cox ${ }^{16}$, Elisabete da Cunha ${ }^{17}$, Emanuele Daddi ${ }^{18}$ (i), \\ Tanio Díaz-Santos ${ }^{7}$, David Elbaz ${ }^{18}$, Jacqueline Hodge ${ }^{3}$ (1) , Hanae Inami ${ }^{19}$, Rob Ivison ${ }^{20,21}$ (10, Olivier Le Fèvree ${ }^{22}$, \\ Benjamin Magnelli ${ }^{12}$ (10), Pascal Oesch ${ }^{23,24}$ (10), Dominik Riechers ${ }^{4,25}$ (1), Ian Smail ${ }^{26}$ (1), Rachel S. Somerville ${ }^{27,28}$, \\ A. M. Swinbank ${ }^{26}$ (1) , Bade Uzgil ${ }^{4,5}$ (1), Paul van der Werf ${ }^{3}$, Jeff Wagg $^{29}$, and Lutz Wisotzki ${ }^{30}$ \\ ${ }^{1}$ Núcleo de Astronomía, Facultad de Ingeniería y Ciencias, Universidad Diego Portales, Av. Ejército 441, Santiago, Chile; manuel.aravenaa@mail.udp.cl \\ ${ }^{2}$ INAF-Osservatorio di Astrofisica e Scienza dello Spazio, via Gobetti 93/3, I-40129, Bologna, Italy \\ ${ }^{3}$ Leiden Observatory, Leiden University, P.O. Box 9513, NL2300 RA Leiden, The Netherland \\ ${ }^{4}$ Max Planck Institute für Astronomie, Königstuhl 17, D-69117 Heidelberg, Germany \\ 5 National Radio Astronomy Observatory, Pete V. Domenici Array Science Center, P.O. Box O, Socorro, NM 87801, USA \\ ${ }^{6}$ Max-Planck-Institut für Radioastronomie, Auf dem Hügel 69, D-53121 Bonn, Germany \\ ${ }^{7}$ Núcleo de Astronomía, Facultad de Ingeniería, Universidad Diego Portales, Av. Ejército 441, Santiago, Chile \\ ${ }^{8}$ Univ. Lyon 1, ENS de Lyon, CNRS, Centre de Recherche Astrophysique de Lyon (CRAL) UMR5574, F-69230 Saint-Genis-Laval, France \\ ${ }^{9}$ Instituto de Astrofísica, Facultad de Física, Pontificia Universidad Católica de Chile Av. Vicuña Mackenna 4860, $782-0436$ Macul, Santiago, Chile \\ ${ }^{10}$ Millennium Institute of Astrophysics (MAS), Nuncio Monseñor Sótero Sanz 100, Providencia, Santiago, Chile \\ ${ }^{11}$ Space Science Institute, 4750 Walnut Street, Suite 205, Boulder, CO 80301, USA \\ ${ }^{12}$ Argelander-Institut für Astronomie, Universität Bonn, Auf dem Hügel 71, D-53121 Bonn, Germany \\ 13 Institut de Recherche en Astrophysique et Planétologie (IRAP), Université de Toulouse, CNRS, UPS, F-31400 Toulouse, France \\ ${ }^{14}$ Joint ALMA Observatory-ESO, Av. Alonso de Córdova, 3104, Santiago, Chile \\ ${ }^{15}$ National Radio Astronomy Observatory, 520 Edgemont Road, Charlottesville, VA, 22903, USA \\ ${ }^{16}$ Institut d'Astrophysique de Paris, 98 bis boulevard Arago, F-75014 Paris, France

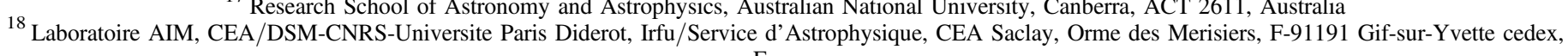 \\ France \\ ${ }^{19}$ Hiroshima Astrophysical Science Center, Hiroshima University, 1-3-1 Kagamiyama, Higashi-Hiroshima, Hiroshima 739-8526, Japan \\ ${ }^{20}$ European Southern Observatory, Karl-Schwarzschild-Strasse 2, D-85748, Garching, Germany \\ ${ }^{21}$ Institute for Astronomy, University of Edinburgh, Royal Observatory, Blackford Hill, Edinburgh EH9 3HJ, UK \\ 22 Aix Marseille Université, CNRS, LAM (Laboratoire d'Astrophysique de Marseille), UMR 7326, F-13388 Marseille, France \\ ${ }^{23}$ Department of Astronomy, University of Geneva, Ch. des Maillettes 51, 1290 Versoix, Switzerland

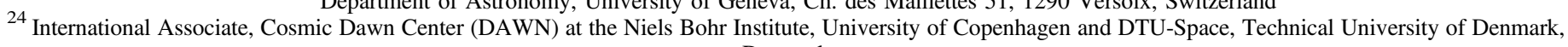 \\ Denmark \\ 25 Cornell University, 220 Space Sciences Building, Ithaca, NY 14853, USA \\ ${ }^{26}$ Centre for Extragalactic Astronomy, Department of Physics, Durham University, South Road, Durham, DH1 3LE, UK
${ }^{27}$ Department of Physics and Astronomy, Rutgers, The State University of New Jersey, 136 Frelinghuysen Road, Piscataway, NJ 08854, USA \\ ${ }^{28}$ Center for Computational Astrophysics, Flatiron Institute, 162 th Avenue, New York, NY 10010, USA \\ ${ }^{29}$ SKA Organization, Lower Withington Macclesfield, Cheshire SK11 9DL, UK \\ ${ }^{30}$ Leibniz-Institut für Astrophysik Potsdam (AIP), An der Sternwarte 16, D-14482 Potsdam, Germany \\ Received 2019 March 19; revised 2019 July 5; accepted 2019 July 10; published 2019 September 11
}

\begin{abstract}
We analyze the interstellar medium properties of a sample of 16 bright CO line emitting galaxies identified in the ALMA Spectroscopic Survey in the Hubble Ultra Deep Field (ASPECS) Large Program. This CO-selected galaxy sample is complemented by two additional CO line emitters in the UDF that are identified based on their MultiUnit Spectroscopic Explorer (MUSE) optical spectroscopic redshifts. The ASPECS CO-selected galaxies cover a larger range of star formation rates (SFRs) and stellar masses compared to literature $\mathrm{CO}$ emitting galaxies at $z>1$ for which scaling relations have been established previously. Most of ASPECS CO-selected galaxies follow these established relations in terms of gas depletion timescales and gas fractions as a function of redshift, as well as the SFR-stellar mass relation ("galaxy main sequence"). However, we find that $\sim 30 \%$ of the galaxies (5 out of 16) are offset from the galaxy main sequence at their respective redshift, with $\sim 12 \%$ ( 2 out of 16 ) falling below this relationship. Some CO-rich galaxies exhibit low SFRs, and yet show substantial molecular gas reservoirs, yielding long gas depletion timescales. Capitalizing on the well-defined cosmic volume probed by our observations, we measure the contribution of galaxies above, below, and on the galaxy main sequence to the total cosmic molecular gas density at different lookback times. We conclude that main-sequence galaxies are the largest contributors to the molecular gas density at any redshift probed by our observations $(z \sim 1-3)$. The respective contribution by starburst galaxies above the main sequence decreases from $z \sim 2.5$ to $z \sim 1$, whereas we find tentative evidence for an increased contribution to the cosmic molecular gas density from the passive galaxies below the main sequence.
\end{abstract}

Key words: galaxies: evolution - galaxies: ISM - galaxies: star formation - galaxies: statistics 


\section{Introduction}

One of the major goals of galaxy evolution studies has been to understand how galaxies transform their gas reservoirs into stars as a function of cosmic time, and how they eventually halt their star formation activity.

An important development has been the discovery that most of the star-forming galaxies show a tight correlation between their stellar masses and star formation rates (SFRs; e.g., Brinchmann et al. 2004; Daddi et al. 2007; Elbaz et al. 2007, 2011; Noeske et al. 2007; Peng et al. 2010; Rodighiero et al. 2010; Whitaker et al. 2012, 2014; Schreiber et al. 2015). Galaxies in this sequence, usually called "main-sequence" (MS) galaxies, would form stars in a steady state for $\sim 1-2$ billion years and dominate the cosmic star formation activity. Galaxies above this sequence, forming stars at higher rates for a given stellar mass, are called "starbursts"; and galaxies below this sequence, are called "passive" or "quiescent" galaxies. The large gas reservoirs necessary to sustain the star-forming activity along the MS would be provided through a continuous supply from the intergalactic medium and minor mergers (Kereš et al. 2005; Dekel et al. 2009). As a consequence, the fundamental galaxy parameters (SFRs, stellar masses, gas fractions, and gas depletion timescales) are found to be closely related at different redshifts. Galaxies above the MS have boosted their SFRs typically through a major merger event (e.g., Kartaltepe et al. 2012).

A critical parameter in the interstellar medium (ISM) characterization has been the specific SFR (sSFR), defined as the ratio between the SFR and stellar mass (SFR/ $M_{\text {stars }}$ ), which for a linear scaling between these parameters denotes how far a galaxy is from the MS population at a given redshift and stellar mass. As a result of observations of gas and dust in starforming galaxies at high redshift in the last decades (for a detailed summary, see Tacconi et al. 2018; Freundlich et al. 2019), current studies indicate that there is an increase of the gas depletion timescales and a decrease in the molecular gas fractions with decreasing redshift $(z \sim 3$ to 1$)$, and that the gas depletion timescales decrease with increasing sSFR (Bigiel et al. 2008; Daddi et al. 2010a, 2010b; Genzel et al. 2010, 2015; Tacconi et al. 2010, 2013, 2018; Saintonge et al. 2011b, 2013, 2016; Leroy et al. 2013; Santini et al. 2014; Sargent et al. 2014; Papovich et al. 2016; Schinnerer et al. 2016; Scoville et al. 2016, 2017; Freundlich et al. 2019; Wiklind et al. 2019). Finally, after galaxies would have formed most of their stellar mass on and above the MS, they would slow down or even halt star formation when they exhausted most of their gas reservoirs (e.g., Peng et al. 2010), bringing them below the MS line.

Observations of the cold molecular gas in high-redshift galaxies have typically relied on transitions of carbon monoxide, ${ }^{12} \mathrm{CO}$ (hereafter $\mathrm{CO}$ ), to infer the existence of large gas reservoirs, as $\mathrm{CO}$ is the second most abundant molecule in the ISM of star-forming galaxies after $\mathrm{H}_{2}$ and given the difficulty in directly detecting $\mathrm{H}_{2}$ (Solomon \& Vanden Bout 2005; Omont 2007; Carilli \& Walter 2013).

While progress has been substantial, there are still potential biases that have so far been little explored. For example, most of the high-redshift galaxies for which observations of molecular gas and dust are available have been preselected from optical and near-IR extragalactic surveys, based on their stellar masses and SFRs estimated from spectral energy distribution (SED) fitting or $\mathrm{UV} / 24 \mu \mathrm{m}$ photometry. Also due to the finite instrumental bandwidth of millimeter interferometers, $\mathrm{CO}$ line studies rely on optical/near-IR redshift measurements. In most cases this means that galaxies need to have relatively bright emission or absorption lines, or display strong features in the continuum. Similarly, galaxy selection based on detections in Spitzer and Herschel far-IR maps, or in ground-based submillimeter observations, will target the most strongly star-forming galaxies, and are in many cases affected by source blending due to the poor angular resolution of these space missions. In turn, this means that such source preselection will select massive galaxies on or above the massive end of the MS.

A complementary approach to the targeted observations has been the so-called "molecular line scan" strategy (Carilli \& Blain 2002; Walter et al. 2014). Here, millimeter/centimeter line observations of an extragalactic "blank-field" are performed using a sensitive interferometer, exploring a significant frequency range (e.g., the full $3 \mathrm{~mm}$ and/or $1 \mathrm{~mm}$ band) over a sizable area of the sky. This essentially provides a large data cube to search for the redshifted emission from $\mathrm{CO}$ emission lines and/or cold dust continuum. Under this approach, galaxies are selected purely based on their molecular gas content. Pioneering observations of the Hubble Deep Field North (HDF-N) with the Plateau de Bureau Interferometer, covering the full $3 \mathrm{~mm}$ band, led to the first estimates of the $\mathrm{CO}$ luminosity functions (LF) at high redshift and the first constraints on the cosmic density of molecular gas (Decarli et al. 2014; Walter et al. 2014). More recently, observations with the Karl Jansky Very Large Array at centimeter wavelengths in the COSMOS field and the HDF-N have allowed to cover larger areas, enabling the characterization of larger samples of gas rich galaxies, and providing tighter constraints on the CO LF and the evolution of the cosmic density of molecular gas (Pavesi et al. 2018; Riechers et al. 2019).

The ALMA Spectroscopic Survey (ASPECS) is the first contiguous molecular survey of distant galaxies performed with ALMA. The ASPECS pilot program targeted a region of 1 $\operatorname{arcmin}^{2}$ of the Hubble Ultra Deep Field (HUDF), scanning the full $3 \mathrm{~mm}$ and $1 \mathrm{~mm}$ bands. This enabled independent line searches in each band (Walter et al. 2016), allowing the investigation of a variety of topics including the characterization of CO-selected galaxies (Decarli et al. 2016a), constraints on the CO LF and cosmic density of molecular gas (Decarli et al. 2016b), derivation of $1 \mathrm{~mm}$ continuum number counts and study of the properties of the faintest dusty galaxies (Aravena et al. 2016b; Bouwens et al. 2016), searches for [C II] line emission at $z>6$ (Aravena et al. 2016c), and derivation of constraints for $\mathrm{CO}$ intensity mapping experiments (Carilli et al. 2016).

The ASPECS program has since been expanded, representing the first extragalactic ALMA large program (LP). ASPECS LP builds upon the observational strategy and the results presented by the ASPECS pilot observations, but extending the covered area of the HUDF from $\sim 1 \operatorname{arcmin}^{2}$ to $5 \operatorname{arcmin}^{2}$, comprising the full area encompassed by the Hubble eXtremely Deep Field (XDF). We here report results based on the $3 \mathrm{~mm}$ data obtained as part of the ASPECS LP.

The ASPECS LP survey strategy and derivation of the CO LF and evolution of the cosmic molecular gas density are presented by Decarli et al. (2019). The line and continuum search techniques, as well as $3 \mathrm{~mm}$ continuum image and 
number counts are presented in González-López et al. (2019). The optical source and redshift identification and global galaxy properties, based on the ultra deep optical/near-IR coverage of the UDF are presented in Boogaard et al. (2019). A theoretical prediction of the cosmic evolution of the CO LF and comparison to the ASPECS measurements are presented in Popping et al. (2019).

In this paper, we analyze the ISM properties of the 16 statistically reliable CO line identifications plus 2 lower significance CO lines identified through optical redshifts, and compare them with the properties of previous targeted $\mathrm{CO}$ observations at high redshift. In Section 2, we briefly summarize the ASPECS LP observations and the ancillary data used in this work. In Section 3, we present the CO line properties. In Section 4, we compare the ISM properties of our ASPECS CO galaxies with standard scaling relations derived from targeted observations of star-forming galaxies. In Section 5, we summarize the main conclusions from this work. Hereafter, we assume a standard $\Lambda \mathrm{CDM}$ cosmology with $H_{0}=70 \mathrm{~km} \mathrm{~s}^{-1} \mathrm{Mpc}^{-1}, \Omega_{\Lambda}=0.7$, and $\Omega_{M}=0.3$.

\section{Observations}

The ASPECS LP uses the same observational strategy followed by the ASPECS pilot survey (Walter et al. 2016), but expanding the covered area to $\sim 5 \operatorname{arcmin}^{2}$. The ASPECS approach is to perform frequency scans over the ALMA bands 3 and 6 (corresponding to the atmospheric bands at $85-115 \mathrm{GHz}$ and $212-272 \mathrm{GHz}$, respectively) and mapping the selected area through mosaics. The overall strategy is to search in this data cube for molecular gas rich galaxies through their redshifted ${ }^{12} \mathrm{CO}$ emission lines entering the ALMA bands. The ASPECS LP band 3 survey setup and data reduction steps are discussed in detail by Decarli et al. (2019). Details about the line search procedures are presented in González-López et al. (2019). For completeness, we repeat the most relevant information for the analysis presented here.

\subsection{ALMA band 3}

ALMA band 3 observations were obtained during Cycle 4 as part of the LP project 2016.1.00324.L. The observations were performed using a 17-point mosaic centered at (R.A., decl. $)=(03: 32: 38.0,-27: 47: 00)$ in the HUDF. We used the spectral scan mode, covering the ALMA band 3, from 84.0 to $115.0 \mathrm{GHz}$ in 5 frequency setups. This strategy yielded an areal coverage of $4.6 \operatorname{arcmin}^{2}$ at $99.5 \mathrm{GHz}$ at the half power beamwidth of the mosaic. Observations were performed in a compact array configuration, C40-3, yielding a synthesized beam of 1 !" $75^{\prime \prime} \times 1$ ". 49 at $99.5 \mathrm{GHz}$.

The data were calibrated and imaged using the CASA software, using an independent procedure, which follows the ALMA pipeline closely. The visibilities were inverted using the TCLEAN task. Since no very bright sources are found in the data cube, we used the "dirty" cubes. The data were rebinned to a channel resolution of $7.813 \mathrm{MHz}$, corresponding to $23.5 \mathrm{~km} \mathrm{~s}^{-1}$ at $99.5 \mathrm{GHz}$. The final cube reaches a sensitivity of $\sim 0.2 \mathrm{mJy}$ beam $^{-1}$ per $23.5 \mathrm{~km} \mathrm{~s}^{-1}$ channel, yielding $5 \sigma \mathrm{CO}$ line sensitivities of $\sim(1.4,2.1,2.3) \times 10^{9} \mathrm{~K} \mathrm{~km} \mathrm{~s}^{-1} \mathrm{pc}^{2}$ for $\mathrm{CO}(2-1), \mathrm{CO}(3-2)$ and $\mathrm{CO}(4-3)$, respectively (Decarli et al. 2019). Our ALMA band 3 scan provides coverage for the redshifted line emission from $\mathrm{CO}(1-0), \mathrm{CO}(2-1), \mathrm{CO}(3-2)$ and $\mathrm{CO}(4-3)$ in the redshift ranges $0.003-0.369,1.006-1.738$,
2.008-3.107, and 3.011-4.475, respectively (Walter et al. 2016; Decarli et al. 2019).

\subsection{CO Sample}

To inspect the data cubes we used the LineSeeker line search routine (González-López et al. 2017). This algorithm convolves the data along the frequency axis with an expected input line width, reporting pixels with signal-to-noise $(\mathrm{S} / \mathrm{N})$ values above a certain threshold. Kernel widths ranging from 50 to $500 \mathrm{~km} \mathrm{~s}^{-1}$ were adopted. The probability of each line candidate of not being due to noise peaks, or Fidelity, $F$, was assessed by using the number of negative line sources in the data cube, with $F=1-N_{\mathrm{Neg}} / N_{\text {Pos }}$. Here, $N_{\text {Neg }}$ and $N_{\text {Pos }}$ correspond to the number of negative and positive emission line candidates with a given $\mathrm{S} / \mathrm{N}$ value in a particular kernel convolution (González-López et al. 2019). We select the sources for which the fidelity is above 0.9. This yields 16 selected line candidates. All of them, except two sources have fidelity values of 1.0. We find that $3 \mathrm{~mm} .15$ and $3 \mathrm{~mm} .16$ have fidelity values of 0.99 and 0.92 , respectively. All these sources are very unlikely to be false positives, based on the statistics presented by González-López et al. (2019). Two other independent line searches were performed using similar algorithms with the findclumps (Decarli et al. 2016a; Walter et al. 2016) and MF3D (Pavesi et al. 2018) codes. All the algorithms coincide in the statistical reliability of these sources. As we mention below, all the selected sources have reliable and matching optical/near-infrared counterparts. The sample of 16 line candidates thus constitutes our primary sample, all of which have $\mathrm{S} / \mathrm{N}>6.4$.

Two additional sources were selected based on the availability of an optical spectroscopic redshift and a matching positive line feature in the ALMA cube at the corresponding frequency. By construction, these sources are selected at lower significance than the CO-selected sources. For more details please refer to Boogaard et al. (2019). This makes up a sample of 18 galaxies detected in $\mathrm{CO}$ emission by the ASPECS program in band-3.

\subsection{Ancillary Data and SEDs}

Our ALMA observations cover roughly the same region as the Hubble XDF. Available data include Hubble Space Telescope (HST) Advanced Camera for Surveys and Wide Field Camera 3 IR data from the HUDF09, HUDF12, and Cosmic Assembly Near-infrared Deep Extragalactic Legacy Survey (CANDELS) programs, as well as public photometric and spectroscopic catalogs (Coe et al. 2006; Xu et al. 2007; Rhoads et al. 2009; McLure et al. 2013; Schenker et al. 2013; Bouwens et al. 2014; Skelton et al. 2014; Momcheva et al. 2015; Morris et al. 2015; Inami et al. 2017). In this study, we make use of this optical and infrared coverage of the XDF, including the photometric and spectroscopic redshift information available from Skelton et al. (2014). The area covered by the ASPECS LP footprint was observed by the Multi-Unit Spectroscopic Explorer (MUSE) Hubble Ultra Deep Survey (Bacon et al. 2017), representing the main optical spectroscopic sample in this area (Inami et al. 2017). The MUSE at the ESO Very Large Telescope provides integral field spectroscopy in the wavelength range $4750-9350 \AA$ of a $3^{\prime} \times 3^{\prime}$ region in the HUDF, and a deeper $1^{\prime} \times 1^{\prime}$ region, which mostly overlaps with the ASPECS field. The MUSE spectroscopic survey 
provides spectroscopic redshifts for optically faint galaxies at the $\sim 30$ mag level, and is thus very complimentary to our ASPECS survey. In addition to the HST coverage, a wealth of optical and infrared coverage from ground-based telescopes is available in this field, including the Spitzer Infrared Array Camera (IRAC) and Multiband Imaging Photometer (MIPS), as well as by the Herschel PACS and SPIRE photometry (Elbaz et al. 2011). From this, we created a master photometric and spectroscopic catalog of the XDF region as detailed in Decarli et al. (2019), which includes $>30$ bands for $\sim 7000$ galaxies, 475 of which have spectroscopic redshifts.

We fit the SED of the continuum-detected galaxies using the high-redshift extension of MAGPHYS (da Cunha et al. 2008, 2015), as described in detail in Boogaard et al. (2019). We use the available broad- and medium-band filters in the optical and infrared regimes, from the $U$ band to Spitzer IRAC $8 \mu \mathrm{m}$, including also the Spitzer MIPS $24 \mu \mathrm{m}$ and Herschel PACS $100 \mu \mathrm{m}$ and $160 \mu \mathrm{m}$. We also include the ALMA 1.2$\mathrm{mm}$ and 3.0-mm data flux densities from Dunlop et al. (2017) and González-López et al. (2019); however, we note that the optical/infrared data have a much stronger weight given the tighter constraints in this part of the spectra. We do not include Herschel SPIRE photometry in the fits because its angular resolution is very poor, being almost the size of our target field for some of the IR bands. For each individual galaxy, we perform SED fits to the photometry fixed at the CO redshift. MAGPHYS employs a physically motivated prescription to balance the energy output at different wavelengths. MAGPHYS delivers estimates for the stellar mass, SFR, dust mass, and IR luminosity. Estimates on the IR luminosity and/or dust mass come from constraints on the dust-reprocessed UV light, which is well sampled by the UV-to-infrared photometry. The derived parameters are listed in Table 2.

\section{Results}

\subsection{CO Measurements}

By construction, the ASPECS CO-based sources presented here are selected through their high significance $\mathrm{CO}$ line detection. The moment- 0 images for each galaxy are created by collapsing the data cube along the frequency axis, considering all the channels within the $99.99 \%$ percentile range of the line profile. Figure 1 shows a combined $\mathrm{CO}$ image of all the moment- 0 maps of these sources, highlighting the location of each of these sources in the field. This map is obtained by coadding all the individual moment-0 line maps, after masking all the pixels with $\mathrm{S} / \mathrm{N}$ below $2.5 \sigma$. Figure 2 shows the $\mathrm{CO}$ spectral profiles, obtained at the peak position of each of the sources (see also Appendix B).

Following González-López et al. (2019), the total CO intensities were derived from the ASPECS band-3 data cube by creating moment- 0 images, collapsing the cube in velocity around the detected $\mathrm{CO}$ lines, and spatially integrating the emission from pixels within a region containing the $\mathrm{CO}$ emission (see González-López et al. 2019, for more details).

All of our CO sources are clearly identified with optical counterparts, as described in detail by Boogaard et al. (2019). While for most of these sources a photometric redshift is enough to provide an identification of the actual $\mathrm{CO}$ line transition and redshift, a large fraction of them is matched with a MUSE spectroscopic redshift. In three cases $(3 \mathrm{~mm} .8$, $3 \mathrm{~mm} .12$, and $3 \mathrm{~mm} .15$ ), the CO line emission can be either

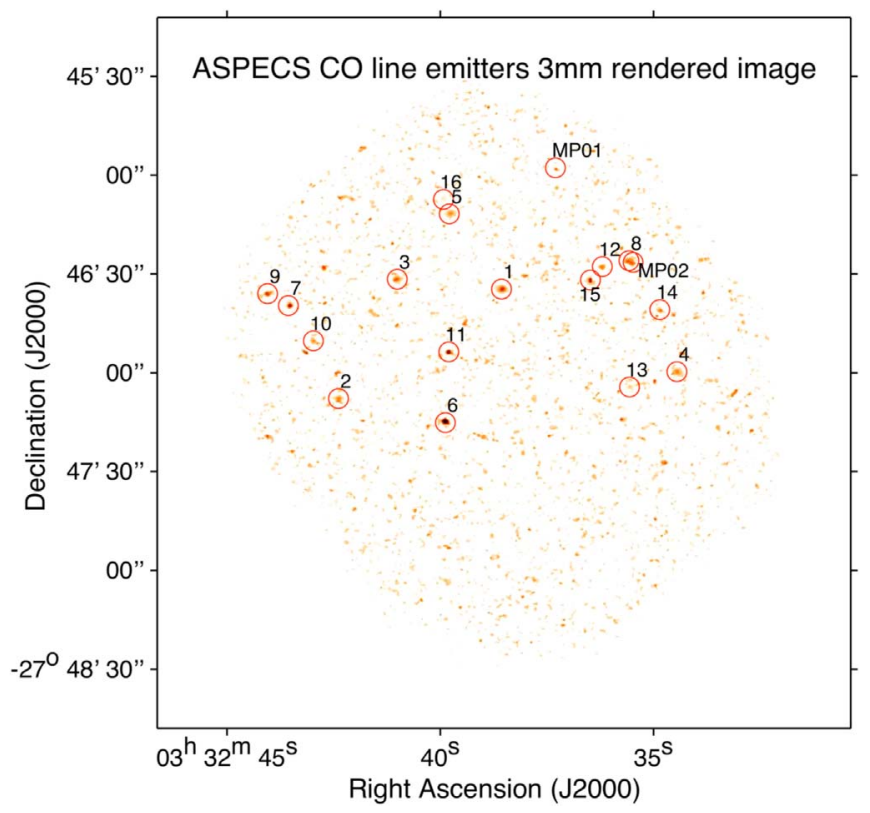

Figure 1. Rendered CO image toward the HUDF, obtained by coadding the individual average $\mathrm{CO}$ line maps around the 16 bright $\mathrm{CO}$-selected galaxies and the 2 lower significance MUSE-based CO sources (labeled MP). Regions with significances below $2.5 \sigma$ in each of the average maps are masked out prior to combination. The location of these individual detections is highlighted by solid circles and their IDs. The tendency of sources to lie in the top two-thirds of the map is likely a combination of clustering and chance, given the sensitivity of the observations is fairly uniform across this region. We note that in this representation of the combined $\mathrm{CO}$ map, some individual images might have larger weight (lower noise) than others, and thus some noise peaks might appear as brighter than other statistically significant sources.

associated with multiple optical sources, due to the higher angular resolution of the optical HST images, or the candidate $\mathrm{CO}$ redshift does not coincide with any of the cataloged photometric or spectroscopic redshifts. In these cases, inspection of the MUSE data cube is critical (Boogaard et al. 2019). For the source $3 \mathrm{~mm} .13$, identified with a $\mathrm{CO}(4-3)$ source at $z \sim 3.601$ we search for a nearby [C I] 1-0 emission line; however, no emission is found at the explored frequency range (see Appendix A). Table 1 lists the CO fluxes, positions, and derived $\mathrm{CO}$ redshifts.

We compute the $\mathrm{CO}$ luminosities, $L_{\mathrm{CO}}^{\prime}$ in units of $\mathrm{K} \mathrm{km} \mathrm{s}^{-1} \mathrm{pc}^{2}$, following Solomon et al. (1997):

$$
L_{\mathrm{CO}}^{\prime}=3.25 \times 10^{7} \nu_{\mathrm{r}}^{-2}(1+z)^{-3} D_{\mathrm{L}}^{2} F_{\mathrm{CO}},
$$

where $\nu_{\mathrm{r}}$ is the rest frequency of the observed CO line, in $\mathrm{GHz}, D_{\mathrm{L}}$ is the luminosity distance at redshift $z$, in $\mathrm{Mpc}$, and $F_{\mathrm{CO}}$ is the integrated CO line flux in $\mathrm{Jy} \mathrm{km} \mathrm{s}^{-1}$. Following Decarli et al. (2016a), we convert the CO luminosities observed at transition $\mathrm{CO}(J \rightarrow J-1)$ to the ground transition $\mathrm{CO}(J=1-0)$ assuming a line brightness temperature ratio, $r_{\mathrm{J} 1}=L_{\mathrm{CO} J \rightarrow J-1}^{\prime} / L_{\mathrm{CO} 1-0}^{\prime}$. From previous observations of massive MS galaxies (Daddi et al. 2015), we adopt $r_{21}=0.76 \pm 0.09, r_{31}=0.42 \pm 0.07$, and $r_{41}=0.31 \pm 0.06$. The uncertainties in $L_{\mathrm{CO}}^{\prime}$ account for the uncertainties in the flux measurements and for the uncertainties due to dispersion in the average $r_{J 1}$ values measured by Daddi et al. (2015). Since the Daddi et al. (2015) observations do not measure the $\mathrm{CO}(4-3)$ lines, but rather $\mathrm{CO}(3-2)$ and $\mathrm{CO}(5-4)$, we extrapolate between those two lines (i.e., we follow the same approach as Decarli et al. 2016b). We note that so far the Daddi 


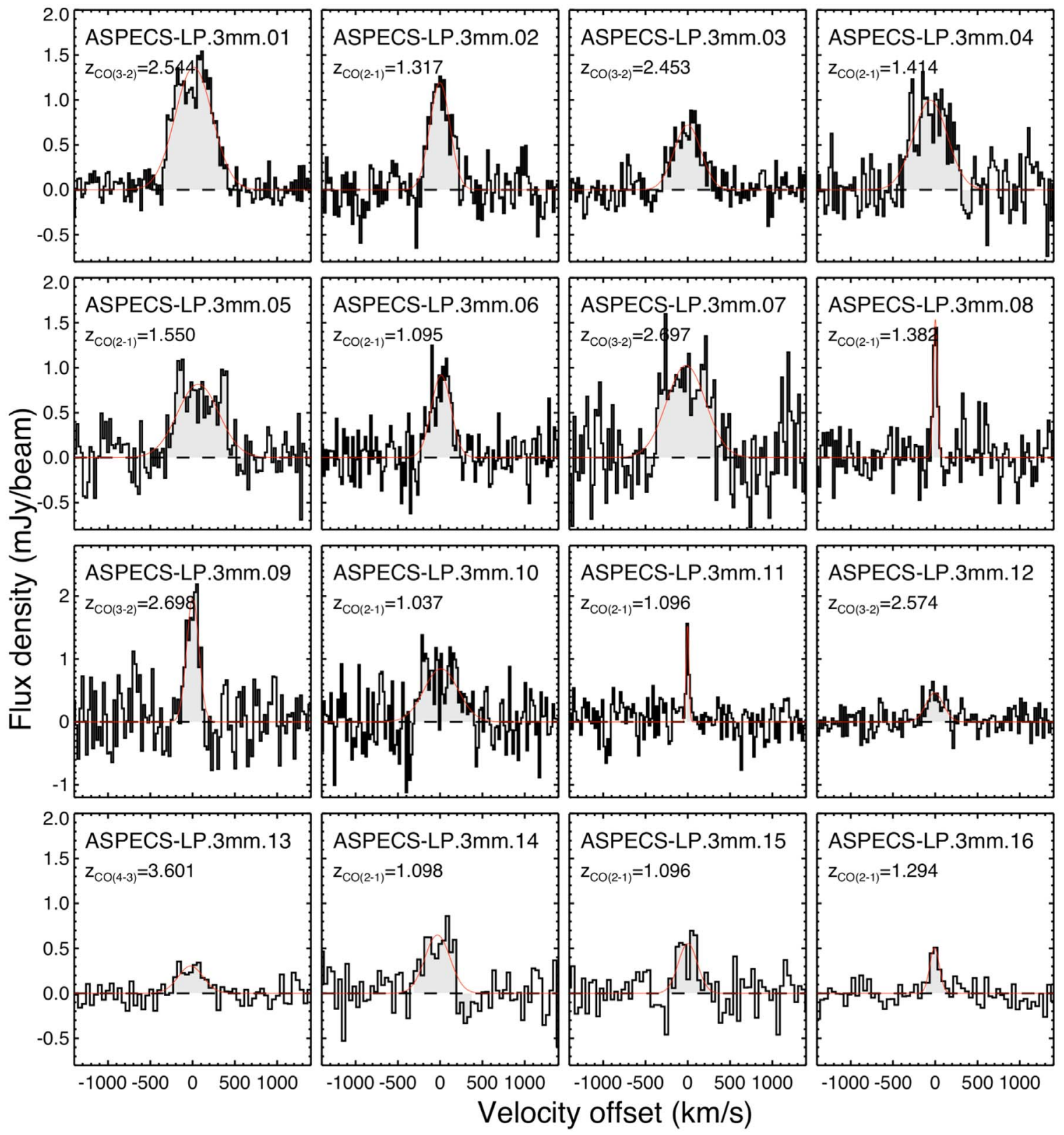

Figure 2. CO line emission profiles obtained from the ALMA $3 \mathrm{~mm}$ data cube, toward the 16 most significant CO-selected detections. The spectra are centered at the identified line, and shown at a width of $7.813 \mathrm{MHz}$ per channel $\left(\sim 25 \mathrm{~km} \mathrm{~s}^{-1}\right)$. For the sources in the bottom row, the spectra have been rebinned by a factor of 2 . The red solid line, represents a one-dimensional Gaussian fit to the profiles. The profiles are obtained by extracting the spectra in the original cube, at the location of the peak position identified in the moment- 0 image. The gray shaded area corresponds to the velocity range used to obtain the moment- 0 images used in Figure 1 .

et al. CO excitation measurements are the only ones available for similar galaxies at these redshifts. These measurements yield excitation values that are intermediate between low-excitation scenarios such as the external part of the disk in the Milky Way and higher-excitation thermalized scenarios in the $J=3-5$ range. This implies that we would not be too far off on either side, if we relax our excitation assumptions. We thus compute the molecular gas masses, in units of $M_{\odot}$, as

$$
M_{\mathrm{H}_{2}}=\alpha_{\mathrm{CO}} L_{\mathrm{CO} 1-0}^{\prime}=\frac{\alpha_{\mathrm{CO}}}{r_{J 1}} L_{\mathrm{CO} J \rightarrow J-1}^{\prime},
$$

where $\alpha_{\mathrm{CO}}$ is the $\mathrm{CO}$ luminosity to gas mass conversion factor in units $M_{\odot}\left(\mathrm{K} \mathrm{km} \mathrm{s}^{-1} \mathrm{pc}^{2}\right)^{-1}$. The value of $\alpha_{\mathrm{CO}}$ has been found to vary from galaxy to galaxy locally, and to depend on various properties of the host galaxies including metallicity and galactic environment (Bolatto et al. 2013). There is a clear dependency of decreasing $\alpha_{\mathrm{CO}}$ values with increasing metallicity (Wilson 1995; Boselli et al. 2002; Leroy et al. 2011; Genzel et al. 2012; Schruba et al. 2012), but there is also a trend with morphology, with lower $\alpha_{\mathrm{CO}}$ for compact starbursts (Downes \& Solomon 1998) compared to extended disks such as the Milky Way. Based on previous observations of massive MS galaxies (Daddi et al. 2010b, 2015; Genzel et al. 2015), we assume a value $\alpha_{\mathrm{CO}}=3.6 M_{\odot}\left(\mathrm{K} \mathrm{km} \mathrm{s}^{-1} \mathrm{pc}^{2}\right)^{-1}$.

To check the reliability of our choice of $\alpha_{\mathrm{CO}}$, we performed an independent computation of this parameter using the 
Table 1

Observed CO Properties

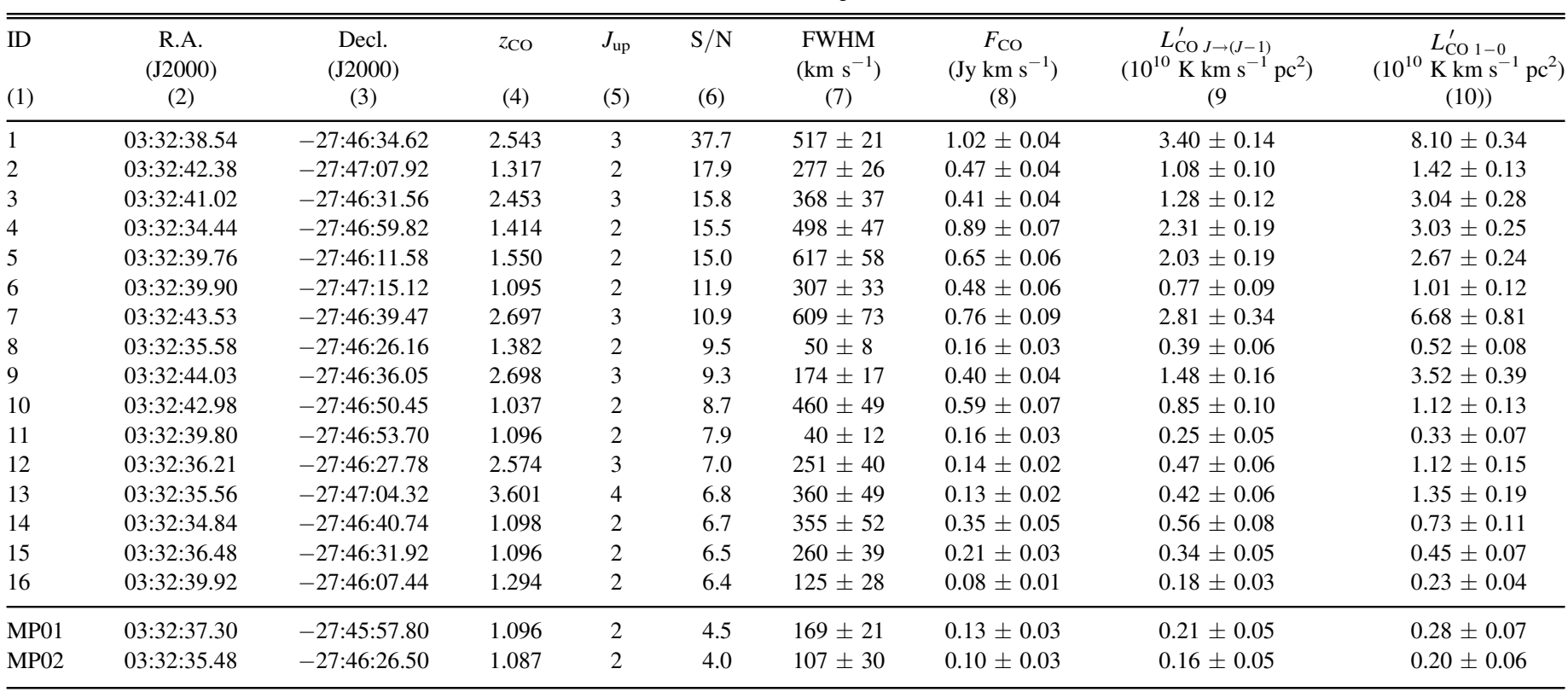

Note. (1) Source ID. ASPECS-LP.3 mm.xx. (2)-(3) CO coordinates of the detection (González-López et al. 2019). (4) CO redshift. (5) Observed CO transition. (6) Signal-to-noise ratio of the detection. (7) CO line full width at half maximum (FWHM). (8) Integrated CO line intensity. (9) CO luminosity of the observed CO transition. (10) $\mathrm{CO}(1-0)$ luminosity, inferred from the observed transitions, under the assumptions mentioned in the main text.

Table 2

ISM Properties of ASPECS CO Galaxies ${ }^{\mathrm{a}}$

\begin{tabular}{|c|c|c|c|c|c|c|c|c|}
\hline ID & $\begin{array}{l}z_{\mathrm{CO}} \\
(2)\end{array}$ & $\begin{array}{c}\mathrm{SFR} \\
\left(M_{\odot} \mathrm{yr}^{-1}\right) \\
(3)\end{array}$ & $\begin{array}{c}M_{\text {stars }} \\
\left(10^{10} M_{\odot}\right) \\
(4)\end{array}$ & $\begin{array}{c}\text { sSFR } \\
\left(\mathrm{Gyr}^{-1}\right) \\
(5)\end{array}$ & $\begin{array}{c}M_{\mathrm{mol}} \\
\left(10^{10} M_{\odot}\right) \\
(6)\end{array}$ & $\begin{array}{l}f_{\text {mol }} \\
(7)\end{array}$ & $\begin{array}{c}t_{\mathrm{dep}} \\
(\mathrm{Gyr}) \\
(8)\end{array}$ & $\begin{array}{c}L_{\mathrm{IR}} \\
\left(10^{11} L_{\odot}\right) \\
(9)\end{array}$ \\
\hline 1 & 2.543 & $233_{-0}^{+0}$ & $2.4_{-0.0}^{+0.0}$ & $9.3_{-0.0}^{+0.0}$ & $29.1 \pm 1.2$ & $12.2_{-0.5}^{+0.5}$ & $1.2_{-0.1}^{+0.1}$ & $80_{-0}^{+0}$ \\
\hline 2 & 1.317 & $11_{-0}^{+3}$ & $15.5_{-1.0}^{+0.7}$ & $0.1_{-0.0}^{+0.0}$ & $5.1 \pm 0.5$ & $0.33_{-0.04}^{+0.03}$ & $4.6_{-0.4}^{+1.1}$ & $3.1_{-0.0}^{+0.5}$ \\
\hline 3 & 2.453 & $68_{-20}^{+19}$ & $5.0_{-0.9}^{+1.0}$ & $1.3_{-0.4}^{+0.6}$ & $10.9 \pm 1.0$ & $2.2_{-0.5}^{+0.5}$ & $1.6_{-0.5}^{+0.5}$ & $8.9_{-2.6}^{+2.6}$ \\
\hline 4 & 1.414 & $61_{-12}^{+3}$ & $18.2_{-2.0}^{+1.3}$ & $0.3_{-0.1}^{+0.0}$ & $10.9 \pm 0.9$ & $0.60_{-0.08}^{+0.07}$ & $1.8_{-0.4}^{+0.2}$ & $9.6_{-1.2}^{+0.2}$ \\
\hline 5 & 1.550 & $62_{-19}^{+6}$ & $32_{-2}^{+1}$ & $0.2_{-0.1}^{+0.0}$ & $9.6 \pm 0.9$ & $0.30_{-0.03}^{+0.03}$ & $1.6_{-0.5}^{+0.2}$ & $11_{-3}^{+1}$ \\
\hline 6 & 1.095 & $34_{-1}^{+0}$ & $3.7_{-0.0}^{+0.1}$ & $0.9_{-0.0}^{+0.0}$ & $3.7 \pm 0.4$ & $1.0_{-0.1}^{+0.1}$ & $1.1_{-0.1}^{+0.1}$ & $3.5_{-0.1}^{+0.0}$ \\
\hline 7 & 2.697 & $187_{-16}^{+38}$ & $12_{-1}^{+2}$ & $1.7_{-0.5}^{+0.3}$ & $24 \pm 3$ & $2.0_{-0.3}^{+0.4}$ & $1.3_{-0.2}^{+0.3}$ & $22_{-2}^{+4}$ \\
\hline 8 & 1.382 & $35_{-5}^{+7}$ & $4.8_{-0.1}^{+0.2}$ & $0.8_{-0.2}^{+0.1}$ & $1.9 \pm 0.3$ & $0.39_{-0.06}^{+0.06}$ & $0.53_{-0.11}^{+0.14}$ & $4.2_{-0.6}^{+0.8}$ \\
\hline 9 & 2.698 & $318_{-34}^{+39}$ & $13_{-1}^{+3}$ & $2.4_{-0.3}^{+0.6}$ & $12.7 \pm 1.4$ & $1.0_{-0.1}^{+0.2}$ & $0.40_{-0.06}^{+0.07}$ & $36_{-4}^{+4}$ \\
\hline 10 & 1.037 & $18_{-1}^{+0}$ & $12 ._{-1}^{+1}$ & $0.2_{-0.0}^{+0.0}$ & $4.0 \pm 0.5$ & $0.33_{-0.05}^{+0.04}$ & $2.2_{-0.3}^{+0.3}$ & $4.5_{-0.4}^{+0.1}$ \\
\hline 11 & 1.096 & $10_{-1}^{+0}$ & $1.5_{-0.1}^{+0.0}$ & $0.7_{-0.1}^{+0.0}$ & $1.2 \pm 0.3$ & $0.78_{-0.18}^{+0.16}$ & $1.2_{-0.3}^{+0.3}$ & $1.1_{-0.1}^{+0.0}$ \\
\hline 12 & 2.574 & $31_{-3}^{+18}$ & $4.4_{-0.5}^{+0.3}$ & $0.7_{-0.0}^{+0.5}$ & $4.1 \pm 0.5$ & $0.93_{-0.16}^{+0.14}$ & $1.3_{-0.2}^{+0.8}$ & $3.4_{-0.3}^{+2.2}$ \\
\hline 13 & 3.601 & $41_{-8}^{+16}$ & $0.6_{-0.1}^{+0.1}$ & $9_{-4}^{+2}$ & $4.9 \pm 0.7$ & $8.5_{-1.9}^{+2.3}$ & $1.2_{-0.3}^{+0.5}$ & $4.2_{-1.0}^{+1.9}$ \\
\hline 14 & 1.098 & $27_{-5}^{+1}$ & $4.1_{-0.5}^{+0.5}$ & $0.6_{-0.00}^{+0.06}$ & $2.6 \pm 0.4$ & $0.65_{-0.13}^{+0.12}$ & $1.0_{-0.2}^{+0.2}$ & $3.4_{-0.8}^{+0.2}$ \\
\hline 15 & 1.096 & $62_{-4}^{+0}$ & $0.5_{-0.0}^{+0.4}$ & $12_{-6}^{+0}$ & $1.6 \pm 0.2$ & $3.2_{-0.5}^{+2.8}$ & $0.26_{-0.04}^{+0.04}$ & $6.9_{-0.0}^{+0.0}$ \\
\hline 16 & 1.294 & $11_{-3}^{+1}$ & $2.1_{-0.1}^{+0.3}$ & $0.5_{-0.1}^{+0.1}$ & $0.8 \pm 0.2$ & $0.39_{-0.07}^{+0.09}$ & $0.73_{-0.22}^{+0.14}$ & $1.0_{-0.3}^{+0.1}$ \\
\hline MP01 & 1.096 & $8_{-2}^{+3}$ & $1.3_{-0.1}^{+0.2}$ & $0.52_{-0.15}^{+0.23}$ & $1.0 \pm 0.2$ & $0.73_{-0.17}^{+0.20}$ & $1.2_{-0.4}^{+0.5}$ & $0_{-80}^{+80}$ \\
\hline MP02 & 1.087 & $25_{-0}^{+0}$ & $2.8_{-0.0}^{+0.0}$ & $0.9_{-0.0}^{+0.0}$ & $0.75 \pm 0.22$ & $0.26_{-0.08}^{+0.08}$ & $0.30_{-0.09}^{+0.09}$ & $2.9_{-0.2}^{+0.7}$ \\
\hline
\end{tabular}

Note.

${ }^{a}$ As noted by Boogaard et al. (2019), formal uncertainties on the derived parameters from the SED fitting are small, systematic uncertainties can be up to 0.3 dex (Conroy 2013). (1) Source ID. ASPECS-LP.3 mm.xx (2) CO redshift. (3)-(5) SFR, stellar mass and specific SFR, derived from MAGPHYS SED fitting. (6) Molecular gas mass, computed from the $\mathrm{CO}$ line luminosity, $L_{\mathrm{CO}}^{\prime}$ and assuming a $\mathrm{CO}$ luminosity to gas mass conversion factor $\alpha_{\mathrm{CO}}=3.6 M_{\odot}\left(\mathrm{K} \mathrm{km} \mathrm{s}^{-1} \mathrm{pc}^{2}\right)^{-1}$. (7) $\mathrm{Gas}$ fraction, defined as $f_{\text {mol }}=M_{\text {mol }} / M_{\text {stars }}$. (8) Molecular gas depletion timescale, $t_{\text {dep }}=M_{\text {mol }} /$ SFR. (9) IR luminosity estimate provided by MAGPHYS SED fitting.

metallicity-dependent approach detailed in Tacconi et al. (2018). This method uses assumptions about the stellar mass-metallicity and the $\alpha_{\mathrm{CO}}$-metallicity relations. Using this prescription, we find very homogeneous metallicity-dependent
$\alpha_{\mathrm{CO}}$ values for the ASPECS CO galaxies. Excluding one source, we find a median of $4.4 M_{\odot}\left(\mathrm{K} \mathrm{km} \mathrm{s}^{-1} \mathrm{pc}^{2}\right)^{-1}$ and a standard deviation of $0.5 M_{\odot}\left(\mathrm{K} \mathrm{km} \mathrm{s}^{-1} \mathrm{pc}^{2}\right)^{-1}$. The excluded source, $3 \mathrm{~mm} .13$, however, is an outlier with $\alpha_{\mathrm{CO}} \sim 13 M_{\odot}$ 

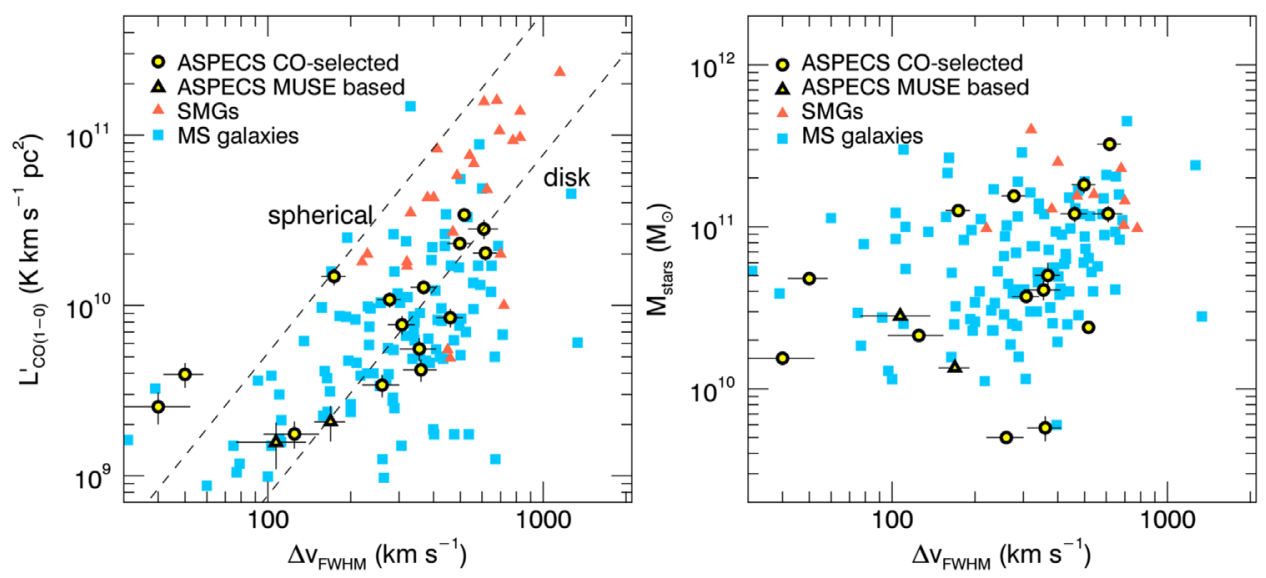

Figure 3. (Left) Estimated $\mathrm{CO}(1-0)$ line luminosities as a function of the line widths $\left(\Delta v_{\mathrm{FWHM}}\right)$ for the ASPECS sources, compared to a compilation of galaxies from the literature detected in CO line emission, including unlensed submillimeter galaxies (Frayer et al. 2008; Coppin et al. 2010; Carilli et al. 2011; Riechers et al. 2011, 2014; Thomson et al. 2012; Bothwell et al. 2013; Hodge et al. 2013; Ivison et al. 2013, 2011; De Breuck et al. 2014) and MS galaxies (Daddi et al. 2010b; Magnelli et al. 2012; Magdis et al. 2012, 2017; Tacconi et al. 2013; Freundlich et al. 2019). The dashed lines represent a simple "virial" functional form for the CO luminosity for a compact starburst and an extended disk (Section 3.2). The actual location of each of these lines depends on the choice of geometry and $\alpha_{\mathrm{CO}}$ factor. (Right) Stellar masses vs. line widths for the ASPECS sources, compared to the literature (where stellar mass estimates are available).

$\left(\mathrm{K} \mathrm{km} \mathrm{s}^{-1} \mathrm{pc}^{2}\right)^{-1}$. Given the close to solar metallicities measured in our $z \sim 1.5$ ASPECS CO sources (Boogaard et al. 2019), and for consistency with other papers in this series, in the following we assume a fixed $\alpha_{\mathrm{CO}}=3.6 M_{\odot}$ $\left(\mathrm{K} \mathrm{km} \mathrm{s}^{-1} \mathrm{pc}^{2}\right)^{-1}$. This will yield $<0.1$ dex differences in the molecular gas mass estimates throughout this study with respect to the metallicity-dependent approach. The following analysis has been checked to remain unchanged if we were assuming a metallicity-dependent $\alpha_{\mathrm{CO}}$ prescription. The computed CO luminosities are listed in Table 1. The corresponding molecular gas masses are listed in Table 2.

\subsection{CO Luminosity versus FWHM}

Following Bothwell et al. (2013), if the CO line emission is able to trace the mass and kinematics of the galaxy, then the $\mathrm{CO}$ luminosity $\left(L_{\mathrm{CO}}^{\prime}\right)$, a tracer of the molecular gas mass and thus proportional to the dynamical mass of the system within the $\mathrm{CO}$ radius (where baryons are expected to be dominant), should be related to the CO line FWHM. A simple parameterization for this relationship is given by (see Harris et al. 2012; Bothwell et al. 2013; Aravena et al. 2016a):

$$
L_{\mathrm{CO}}^{\prime}=C\left(\frac{R}{\alpha_{\mathrm{CO}} G}\right)\left(\frac{\Delta v_{\mathrm{FWHM}}}{2.35}\right)^{2},
$$

where $R$ is the $\mathrm{CO}$ radius in units of kpc, $\Delta v_{\mathrm{FWHM}}$ is the line FWHM in $\mathrm{km} \mathrm{s}^{-1}, \alpha_{\mathrm{CO}}$ is the CO luminosity to molecular gas mass conversion factor in units of $M_{\odot}\left(\mathrm{K} \mathrm{km} \mathrm{s}^{-1} \mathrm{pc}^{2}\right)^{-1}$, and $G$ is the gravitational constant, and $C$ is a constant that depends on the source geometry and inclination (Erb et al. 2006; Bothwell et al. 2013). A similar argument follows for the possible relation between stellar mass and line FWHM.

Figure 3 (left) shows the relationship between the $\mathrm{CO}$ luminosities and the line FWHM for our ASPECS CO galaxies, compared to a compilation of high-redshift galaxies detected in CO line emission from the literature. This includes a sample of unlensed submillimeter galaxies (Frayer et al. 2008; Coppin et al. 2010; Carilli et al. 2011; Riechers et al. 2011, 2014; Thomson et al. 2012; Bothwell et al. 2013; Hodge et al. 2013; Ivison et al. 2013, 2011; De Breuck et al. 2014) and $z>1 \mathrm{MS}$ galaxies (Daddi et al. 2010b; Magdis et al. 2012, 2017; Magnelli et al. 2012; Tacconi et al. 2013). CO line luminosities for MS galaxies have been corrected down to $\mathrm{CO}(1-0)$ using the line ratios mentioned above. All the submillimeter galaxies shown have observations of either $\mathrm{CO}(1-0)$ or $\mathrm{CO}(2-1)$ available, and no correction has been applied in these cases. Also shown in Figure 3, are the parameterization of the $L_{\mathrm{CO}}^{\prime}$ versus FWHM relationship for two representative cases including a disk galaxy model, with $C=2.1, R=4 \mathrm{kpc}$, and $\alpha_{\mathrm{CO}}=4.6 M_{\odot}\left(\mathrm{K} \mathrm{km} \mathrm{s}^{-1} \mathrm{pc}^{2}\right)^{-1}$; and a isotropic (spherical) source, with $C=5, \quad R=2 \mathrm{kpc}, \quad$ and $\quad \alpha_{\mathrm{CO}}=0.8 M_{\odot}$ $\left(\mathrm{K} \mathrm{km} \mathrm{s}^{-1} \mathrm{pc}^{2}\right)^{-1}$. A positive correlation is seen between $L_{\mathrm{CO}}^{\prime}$ and the line FWHM, as already found in previous studies (e.g., Harris et al. 2012; Bothwell et al. 2013; Aravena et al. 2016a). The scatter in this plot is driven by the different CO sizes $(R)$ and inclinations among sources, as well as the choices of $\alpha_{\mathrm{CO}}$ and line ratios. Interestingly, most of the ASPECS CO sources seem to cluster around the "disk" model line and appear to follow a preferred geometry. Similarly, most submillimeter galaxies appear to lie closer to the "spherical" model line. However, this depends on the choice of parameters for the plotted models (a spherical model would also be able to pass through the ASPECS points). Inspection of the optical images (see Appendix C) show that the galaxies' morphologies are complex (see also Boogaard et al. 2019). Instead, this could either hint toward a possible homogeneity of the ASPECS galaxies in terms of their geometry and $\alpha_{\mathrm{CO}}$ factors or just a conspiracy of these. Interestingly, two sources, $3 \mathrm{~mm} .8$ and $3 \mathrm{~mm} .11$, show very narrow line widths (40 and $50 \mathrm{~km} \mathrm{~s}^{-1}$, respectively) for their expected $L_{\mathrm{CO}}^{\prime}$. Inspection of the $H S T$ images (see Appendix C) shows that these galaxies are very likely face-on, and thus the reason for such narrow line widths.

Figure 3 (right) shows the stellar mass versus the $\mathrm{CO}$ line FWHM. Among the $\mathrm{CO}$ sources from the literature, only those with a stellar mass measurement available are shown. More scatter is apparent in this case, arguing for a relative disconnection between the stellar and molecular components. However, the intrinsic uncertainties and differences in the computation of stellar masses makes this difficult to study with the current data. 


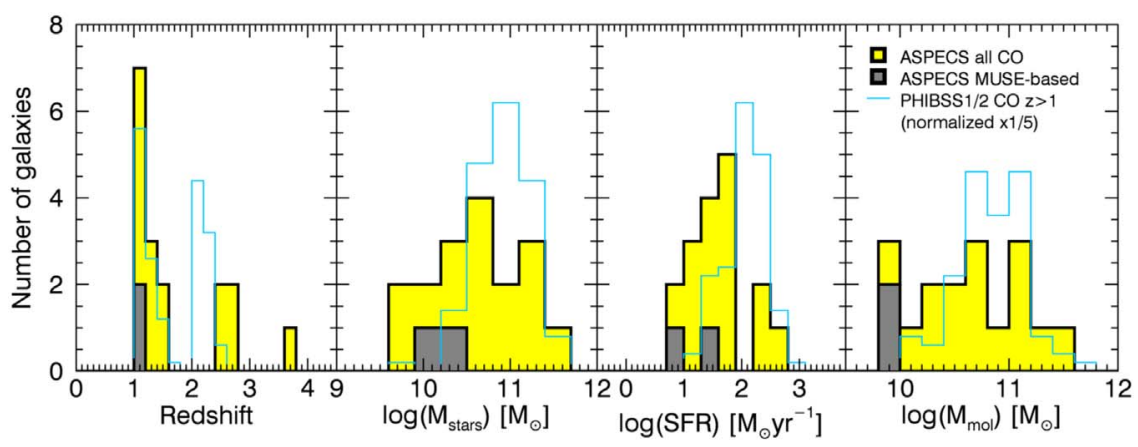

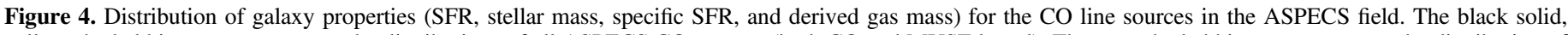

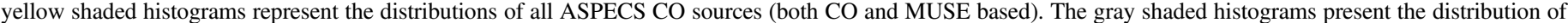

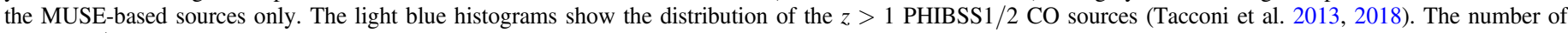

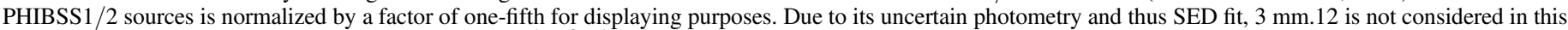

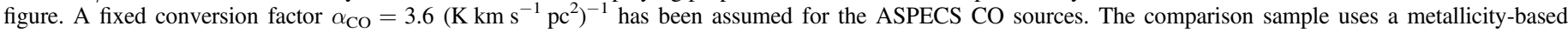
prescription for this parameter.

\section{Analysis and Discussion}

\subsection{CO-selected Galaxies in Context}

The ASPECS CO survey redshift selection function for $\mathrm{CO}$ line detection is roughly limited to galaxies at $z>1$, with a small gap at $z=1.78-2.00$. While it is also possible to detect $\mathrm{CO}(1-0)$ for galaxies at $z<0.4$, the volume surveyed is too small to provide enough statistics.

To put our galaxies into context with respect to previous ISM observations, we compare the properties of the ASPECS $\mathrm{CO}$ galaxies with the compilation published as part of the "Plateau de Bureau High- $z$ Blue Sequence Survey," PHIBBS (Tacconi et al. 2013) and PHIBSS2 (Tacconi et al. 2018; Freundlich et al. 2019). This provides the largest compilation to date of targeted molecular gas mass measurements from $\mathrm{CO}$ line observations, $1 \mathrm{~mm}$ dust photometry and far-infrared SEDs for 1444 galaxies selected from different extragalactic fields (Gao \& Solomon 2004; Greve et al. 2005; Tacconi et al. 2006, 2008; Graciá-Carpio et al. 2008; Graciá-Carpio 2009; Daddi et al. 2010b; Riechers et al. 2010; Tacconi et al. 2010, 2013; Combes et al. 2011, 2013; Saintonge et al. 2011a, 2011b, 2013, 2016, 2017; García-Burillo et al. 2012; Magdis et al. 2012; Magnelli et al. 2012, 2014; Bauermeister et al. 2013; Bothwell et al. 2013; Santini et al. 2014; Béthermin et al. 2015; Genzel et al. 2015; Silverman et al. 2015; Tadaki et al. 2015, 2017; Aravena et al. 2016b; Barro et al. 2016; Berta et al. 2016; Decarli et al. 2016b; Scoville et al. 2016; Schinnerer et al. 2016; Dunlop et al. 2017). The full compilation contains galaxies selected from various different observations and surveys, and thus with different selection functions (Tacconi et al. 2018). To provide a meaningful comparison, we restrict this sample to sources observed as part of the PHIBSS and PHIBSS2 surveys only, detected in CO line emission at $z>1$ (i.e., excluding dust continuum measurements) from Tacconi et al. (2018). This yields a sample of 87 PHIBSS $1 / 2$ CO sources at $z>1$, compared to the 18 ASPECS CO sources, spanning a significant range of properties $\left(\mathrm{SFR} \sim 10-1000 M_{\odot} \mathrm{yr}^{-1}\right.$ and $\left.M_{\text {stars }}=10^{9.5}-10^{11.8} M_{\odot}\right)$.

Given the different nature of the ASPECS survey compared to targeted observations, it is interesting to check how different the ASPECS selection is in terms of basic galaxy parameters. Figure 4 shows the distribution of redshift, stellar mass, SFR, and $\mathrm{CO}$-derived gas masses for all ASPECS CO galaxies, as well as the MUSE-based CO sample, compared with the normalized distribution of $z>1$ PHIBSS $1 / 2 \mathrm{CO}$ galaxies (a normalization factor of one-fifth has been used).

Except for the redshift, these parameters show different distributions for the ASPECS CO galaxies when compared to the $z>1$ PHIBSS1/2 CO galaxies. The ASPECS CO galaxies span a range of two orders of magnitude in stellar mass and three orders of magnitude in SFR. The ASPECS CO galaxies' distributions tend to have lower stellar masses and lower SFRs, with median values of $\sim 10^{10.6} M_{\odot}$ and $35 M_{\odot} \mathrm{yr}^{-1}$, respectively, whereas the bulk of the $z>1$ PHIBSS $1 / 2 \mathrm{CO}$ galaxies have median stellar masses and SFRs of $10^{10.8} M_{\odot}$ and $\sim 100 M_{\odot} \mathrm{yr}^{-1}$, respectively. While there are a few literature galaxies with stellar masses below $10^{10.2} M_{\odot}$, a larger fraction of ASPECS CO galaxies are located in this range (4 out of 18). We find a clear difference in SFRs between our galaxies and the $z>1$ PHIBSS1/2 CO sample, with all except three ASPECS CO galaxies lying below $\sim 100 M_{\odot} \mathrm{yr}^{-1}$ and the bulk of the PHIBSS1/2 CO galaxies above this value. Similarly, while almost none of the galaxies in the comparison sample are found with SFR $<25 M_{\odot} \mathrm{yr}^{-1}, 5$ out of the 18 ASPECS CO sources are found in this range. Furthermore, the ASPECS CO galaxies tend to have a flatter distribution of molecular gas masses and some of them show lower values than the PHIBSS1/2 CO galaxies. Since only part of this can be attributed to differences in the assumed $\alpha_{\mathrm{CO}}$ factors (as the PHIBSS1/2 survey assumes a metallicity/stellar mass dependent $\alpha_{\mathrm{CO}}$ ), this might reflect differences in parameter space between these surveys, i.e., the lower stellar masses and SFRs inherent to our survey.

To quantify these differences between the ASPECS CO and the PHIBSS $1 / 2 \mathrm{CO} z>1$ samples, we computed the two sided Kolmogorov-Smirnov (KS) statistic, which yields the probability that two data sets are drawn from the same distribution. We find KS probabilities of $0.05,2.3 \times 10^{-4}$, and 0.06 for the stellar mass, SFR, and molecular gas mass, respectively. These low values of the KS probability for the stellar mass and SFR distributions point to the differences in the selection between the ASPECS and PHIBSS1/2 surveys, because the latter explicitly did not select galaxies with low SFRs.

Figure 5 shows the location of the ASPECS CO galaxies in the SFR versus stellar mass plane, compared to the $z>1$ PHIBSS1/2 CO galaxies. The ASPECS galaxies are depicted 


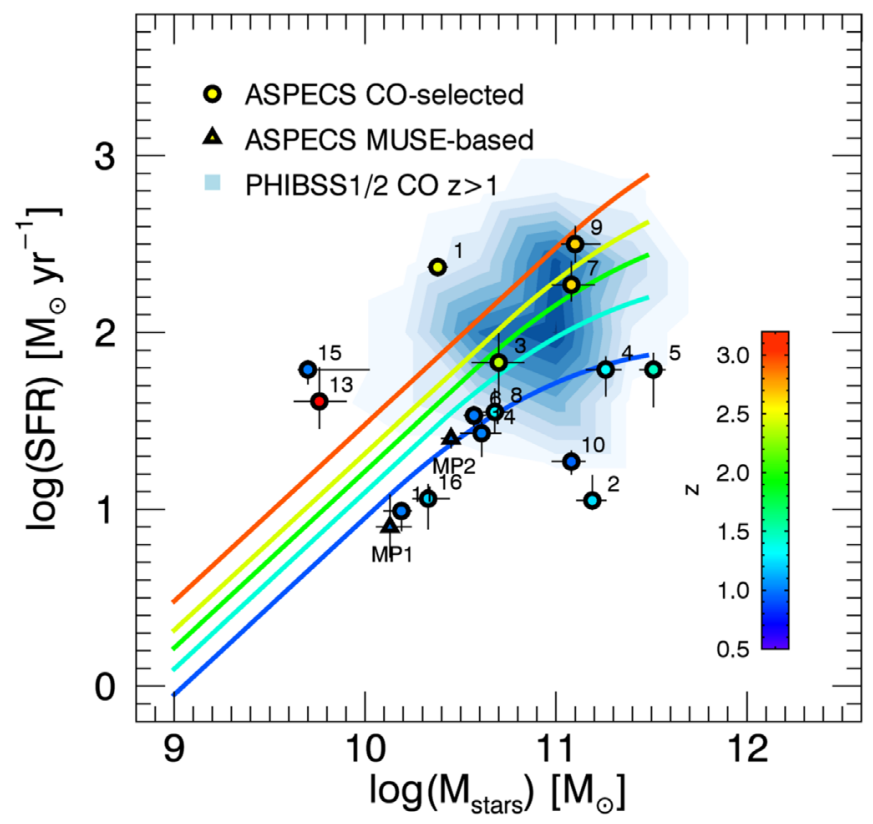

Figure 5. SFR vs. stellar mass diagram for the ASPECS CO sources, compared to PHIBSS1/2 CO sources at $z>1$. The PHIBSS1/2 galaxies are represented by the blue contours. The solid lines represent the observational relationships between SFR and stellar mass at different redshifts derived by Schreiber et al. (2015). These redshifts are denoted in different colors as shown by the color bar to the right. Three of the ASPECS CO-selected galaxies lie $>0.4$ dex below the MS at their respective redshifts $(3 \mathrm{~mm} .2$ and $3 \mathrm{~mm} .10)$.

by large circles and triangles, color-coded to denote their redshifts. Also shown, are the observational relationships derived for the MS galaxies as a function of redshift (Schreiber et al. 2015). We choose to use the Schreiber et al. (2015) MS relationships as a comparison because this prescription is tunable to a specific redshift, produces curves that are similar to those used in other studies (e.g., Speagle et al. 2014; Whitaker et al. 2014), and reproduces the location of the PHIBSS1/2 sources in the MS plane well. A complementary view of the SFR versus stellar mass plot is shown in Figure 6, which presents the sSFR as a function of the stellar mass. The right panel in particular shows the sSFR normalized by the expected sSFR value of the MS (i.e., the offset from the MS). The sSFR of each galaxy is normalized by the expected sSFR value of the MS at the galaxies' redshift and stellar mass, using the MS prescription presented by Schreiber et al. (2015).

Aside from the larger parameter space explored by the ASPECS survey, as mentioned above, we find two galaxies that are significantly below the MS of star-forming galaxies at their respective redshift: $3 \mathrm{~mm} .2$ and $3 \mathrm{~mm} .10$, corresponding to $12.5 \%$ of the CO-selected sample. These galaxies would be classified as "quiescent" galaxies, as their sSFRs are a factor of at least $\sim 0.4$ dex below the value of the MS of galaxies at each particular redshift for a fixed stellar mass. Conversely, in three cases $(3 \mathrm{~mm} .1,3 \mathrm{~mm} .13$, and $3 \mathrm{~mm} .15)$ the location of the sources on this plot makes them consistent with "starbursts," lying 0.4 dex above the MS, and corresponding to $18.7 \%$ of the CO-selected sample. This implies that $\sim 30 \%$ of the COselected sample corresponds to galaxies off the MS. Note that this would still be valid if we consider systematic uncertainties between different calibrations of the MS as a selection of the MS lines. However, differences in the methods used to compute the SFRs and stellar masses by different studies (e.g., Whitaker et al. 2014; Schreiber et al. 2015) compared to the MAGPHYS SED fitting method used here can bring our "quiescent" sources closer to the respective MS lines (e.g., Mobasher et al. 2015). We refer the reader to Boogaard et al. (2019) for a more detailed discussion on this subject.

Figure 7 shows the measured SFRs and CO-derived gas masses for the ASPECS CO galaxies compared to the PHIBSS1/2 CO $z>1$ sample. Dashed lines represent the location of constant depletion timescales $\left(t_{\text {dep }}\right.$; see below for the definition of this parameter). Despite the differences between the ASPECS sources and the PHIBSS1/2 CO $z>1$ sample shown in Figures 4 and 5, the majority of the ASPECS galaxies follow relatively tightly the $t_{\text {dep }} \sim 1$ Gyr line in the SFR $-M_{\text {mol }}$ plot (see Figure 8). This is consistent with the location of the bulk of PHIBSS1/2 CO $z>1$ galaxies, which lie just above this line. Only one ASPECS source, $3 \mathrm{~mm} .2$, tends to lie significantly below this trend, closer to the $t_{\mathrm{dep}}=10 \mathrm{Gyr}$ curve.

Interestingly, we find that the galaxy with the largest offset below the MS line in Figure 5, $3 \mathrm{~mm} .2$, appears to have a significant reservoir of molecular gas $\left(>10^{10} M_{\odot}\right)$, which would be able to sustain star formation for about 5 Gyr at the current rate (Figure 7). This could be interpreted in the sense that this galaxy might have just recently left the MS of starforming galaxies and/or might have recently replenished its molecular gas reservoir. Conversely, the starburst galaxies $3 \mathrm{~mm} .9$ and $3 \mathrm{~mm} .15$ are consistent with short gas depletion timescales $(<1 \mathrm{Gyr})$ as typically found in these kinds of galaxies.

\subsection{Gas Depletion Timescales and Gas Fractions}

The molecular gas depletion timescale is defined as the time needed to exhaust the current molecular gas reservoir at the current level of star formation in a galaxy. In the absence of feedback mechanisms (inflows/outflows) the consumption of the molecular gas is driven by star formation, and thus the gas depletion timescale can be defined as $t_{\text {dep }}=M_{\text {mol }} / \mathrm{SFR}$. Similarly, the gas fraction corresponds to a measurement of how much of the baryonic mass of the galaxy is in the molecular form. This parameter is typically defined as $f_{\text {gas }}=M_{\text {mol }} /\left(M_{\text {mol }}+M_{\text {stars }}\right)$. For this work, we use a simpler quantity, the molecular gas ratio, defined as $\mu_{\mathrm{mol}}=M_{\mathrm{mol}} /$ $M_{\text {stars }}$. Current measurements based on targeted $\mathrm{CO}$ and dust observations of star-forming galaxies indicate that both parameters, $t_{\mathrm{dep}}$ and $f_{\mathrm{gas}}$ (or $\mu_{\mathrm{mol}}$ ), follow clear scaling relations with redshift, sSFR, and stellar mass (Scoville et al. 2017; Tacconi et al. 2018). These studies indicate that the gas depletion timescales evolve moderately with redshift, following $\propto(1+z)^{\alpha}$. The value of $\alpha$ has been found to be -0.62 from observational studies (e.g., Tacconi et al. 2013, 2018), while theoretical studies suggest $\alpha=-1.5$ (Davé et al. 2012). The sSFR follows a steeper evolution with redshift with sSFR $\propto(1+z)^{\beta} M_{\text {stars }}^{-0.1}$, with $\beta$ between $5 / 3$ and 3 (Lilly et al. 2013). Due to the close relationship between these parameters, $\mu_{\mathrm{mol}}=t_{\mathrm{dep}} \mathrm{sSFR}$ or $f_{\mathrm{mol}}=\left[1+\left(t_{\mathrm{dep}} \mathrm{SSFR}\right)^{-1}\right]^{-1}$, the molecular gas fraction is thus predicted to follow a much stronger evolution with $f_{\mathrm{mol}} \propto(1+z)^{1.8-2.5}$. To match up the mild evolution of molecular gas depletion timescales with the evolution of the molecular gas ratios or fractions, galaxies might need high accretion rates (Scoville et al. 2017). While these scaling relations have been successful to describe the properties of star-forming galaxies preselected from optical/near-IR surveys, it is not clear to 

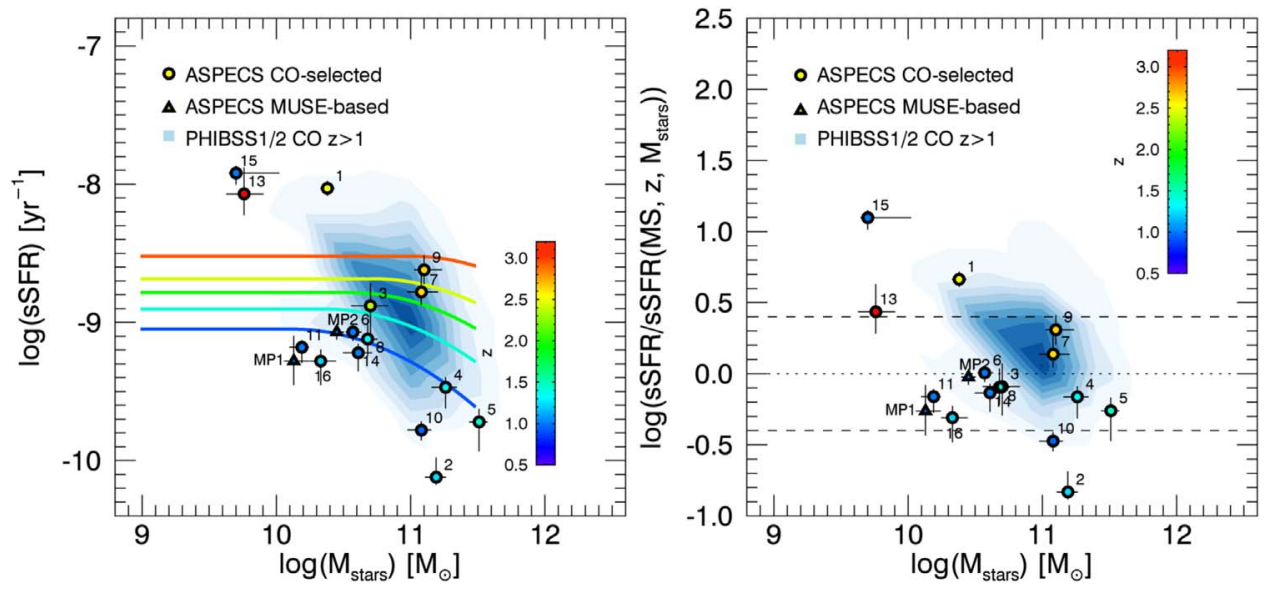

Figure 6. (Left) Specific SFR vs. stellar mass diagram for the ASPECS CO sources, compared to $z>1$ PHIBSS1/2 CO sources (Tacconi et al. 2013, 2018). (Right) Specific SFR (normalized by the value of the sSFR expected for the MS, which is a function of the redshift and stellar mass) vs. stellar mass diagram for the ASPECS CO sources, compared to $z>1$ PHIBSS1/2 CO sources. In both panels, the PHIBSS1/2 galaxies are represented by the blue contours. In the left panel, the solid lines represent the observational relationships between SFR (or sSFR) and stellar mass at different redshifts derived by Schreiber et al. (2015). These redshifts are denoted in different colors as shown by the color bar to the right. In the right panel, the dotted line represents the location of the MS, while the dashed lines represent the location of sources at +0.4 and -0.4 dex from the MS. Two of the ASPECS CO-selected galaxies lie $>0.4$ dex below the MS at their respective redshifts ( 3 mm. 2 and $3 \mathrm{~mm} .10)$.

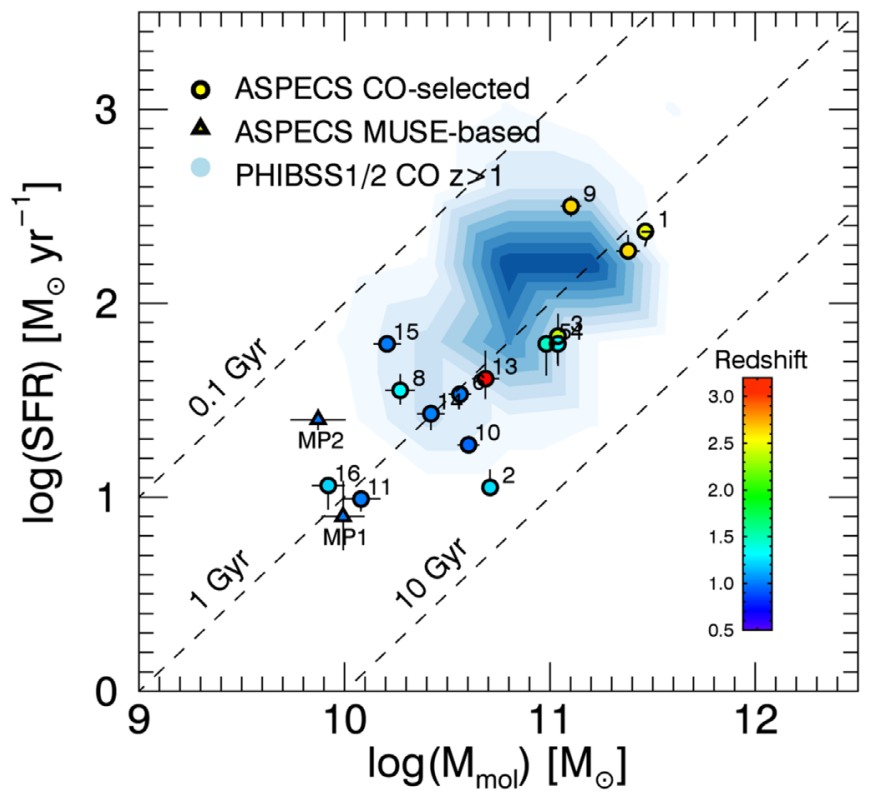

Figure 7. SFR vs. $M_{\text {mol }}$ for the ASPECS CO galaxies compared to the $z>1$ PHIBSS1/2 CO sources (Tacconi et al. 2013, 2018), represented by blue contours as in Figure 5. The dashed lines represent curves of constant $t_{\text {dep }}$ at $0.1,1$, and 10 Gyr. A fixed conversion factor $\alpha_{\mathrm{CO}}=3.6\left(\mathrm{~K} \mathrm{~km} \mathrm{~s}^{-1} \mathrm{pc}^{2}\right)^{-1}$ has been assumed for the ASPECS CO sources. The comparison sample uses a metallicity-based prescription for this parameter. Typical values will range between $\alpha_{\mathrm{CO}}=2-5\left(\mathrm{~K} \mathrm{~km} \mathrm{~s}^{-1} \mathrm{pc}^{2}\right)^{-1}$ for the ASPECS CO sources.

what level they extend to the CO-selected galaxies presented in this study.

Figure 8 depicts the distributions of $t_{\mathrm{dep}}$ and $\mu_{\mathrm{mol}}$ of the ASPECS CO galaxies compared to the $z>1$ PHIBSS $1 / 2$ CO galaxies. The range of the distributions of $t_{\mathrm{dep}}$ for both samples appears similar, although the ASPECS CO galaxies seem to have systematically higher $t_{\mathrm{dep}}$. This difference could be driven by the lower SFRs in the ASPECS sources and in principle this could be driven by the systematic differences in the SED fitting methods (Mobasher et al. 2015). However, we should note that

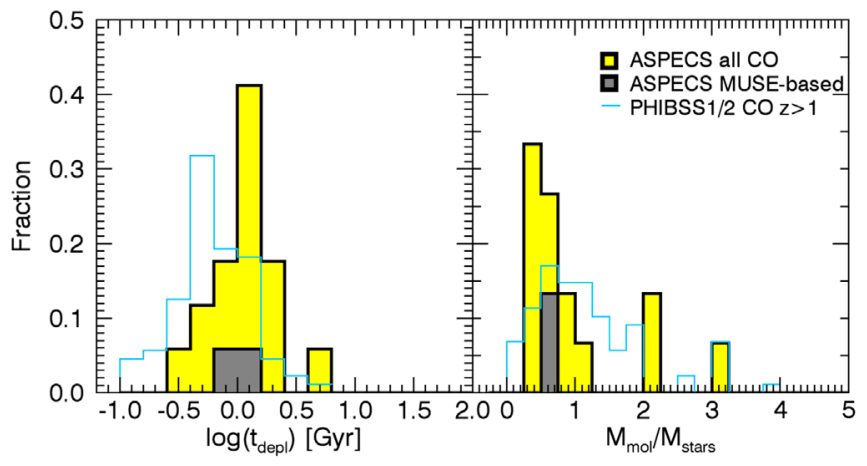

Figure 8. Distribution of derived ISM properties (gas depletion timescale and gas fraction) for the CO line sources in the ASPECS field. The black solid, yellow shaded histogram represents the distributions of all ASPECS sources (both CO and MUSE based). The gray shaded histogram shows the distribution of the MUSE-based sources only. The light blue histograms show the distribution of $z>1$ PHIBSS1/2 CO sources (Tacconi et al. 2013, 2018). Due to its uncertain counterpart photometry, $3 \mathrm{~mm} .12$ is not considered in this figure. Sources $3 \mathrm{~mm} .1$ and $3 \mathrm{~mm} .13$ have high values of $M_{\text {mol }} / M_{\text {stars }}$ falling outside the range covered by this figure. A fixed conversion factor $\alpha_{\mathrm{CO}}=3.6$ $\left(\mathrm{K} \mathrm{km} \mathrm{s}^{-1} \mathrm{pc}^{2}\right)^{-1}$ has been assumed for the ASPECS CO sources. The comparison sample uses a metallicity-based prescription for this parameter. Typical values will range between $\alpha_{\mathrm{CO}}=2-5\left(\mathrm{~K} \mathrm{~km} \mathrm{~s}^{-1} \mathrm{pc}^{2}\right)^{-1}$ for the ASPECS CO sources.

some of the ASPECS CO galaxies have systematically lower gas masses. This could be only partly driven by the different prescriptions used for the $\alpha_{\mathrm{CO}}$ conversion factor between the different samples, because the distributions of molecular gas masses mostly overlap (Figure 4). Conversely, the distributions of $\mu_{\mathrm{mol}}$ appear similar, covering identical ranges. A KS test comparing the distributions of $\mu_{\mathrm{mol}}$ and $t_{\mathrm{dep}}$ yields probabilities of 0.33 and 0.0012 , respectively, indicating that the ASPECS $\mathrm{CO}$ sources follow a different $t_{\text {dep }}$ distribution than the PHIBSS $1 / 2 \mathrm{CO} z>1$ sample.

Figure 9 shows the standard scaling relation between $t_{\mathrm{dep}}$ and sSFR for the ASPECS CO galaxies, compared to the PHIBSS1/2 CO $z>1$ sample. While the distribution of ASPECS galaxies appears considerably wider in this plane than 

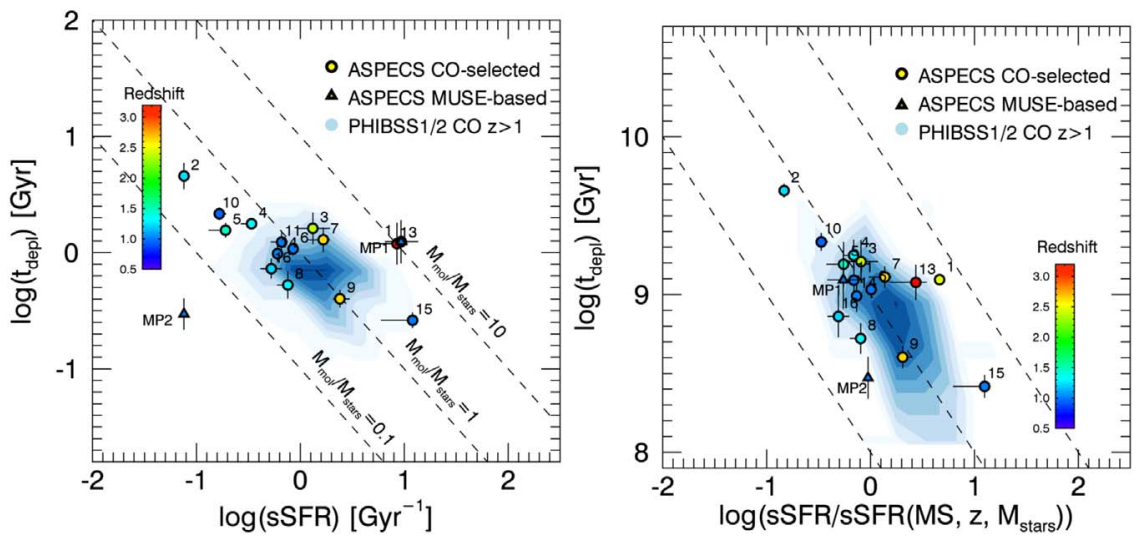

Figure 9. Molecular gas depletion timescale $\left(t_{\text {dep }}\right)$ as a function of the specific SFR for the ASPECS CO galaxies. In both panels, the background blue contour levels represent the distribution of $z>1$ PHIBSS1/2 CO galaxies (Tacconi et al. 2013, 2018), and the coloring of each ASPECS source represents its respective redshift. The left panel shows $t_{\mathrm{dep}}$ as a function of sSFR. Here the dashed lines represent curves of fixed gas fraction $\left(M_{\mathrm{mol}} / M_{\mathrm{starc}}\right)$. The right panel shows the sSFR normalized by the value of the sSFR expected for the MS (which is a function of the redshift and stellar mass) from Schreiber et al. (2015). In this case, the dashed lines are shown only for visualization purposes. A fixed conversion factor $\alpha_{\mathrm{CO}}=3.6\left(\mathrm{~K} \mathrm{~km} \mathrm{~s}^{-1} \mathrm{pc}^{2}\right)^{-1}$ has been assumed for the ASPECS CO sources. The comparison sample uses a metallicity-based prescription for this parameter. Typical values will range between $\alpha_{\mathrm{CO}}=2-5 M_{\odot}\left(\mathrm{K} \mathrm{km} \mathrm{s}^{-1} \mathrm{pc}^{2}\right)^{-1}$ for the ASPECS CO sources.
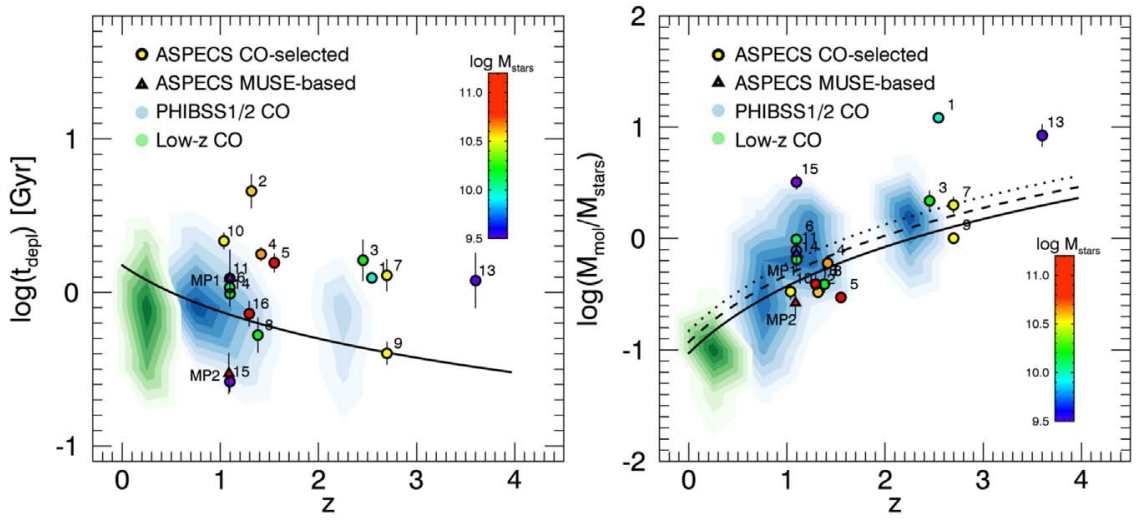

Figure 10. Evolution of the $t_{\mathrm{depl}}$ and $f_{\mathrm{mol}}=M_{\mathrm{mol}} / M_{\mathrm{stars}}$ with redshift. The background blue contour levels represent the distribution of galaxies from the PHIBSS1/2 compilation (Tacconi et al. 2013, 2018). As a reference in redshift, we also show as green contours the distribution of galaxies detected in CO line emission at $z<0.5$ from the PHIBSS1/2 compilation (e.g., from xCOLDGASS, GOALS, and EgNOG surveys). The solid lines show the expected evolution of $t_{\mathrm{dep}}$ and $f_{\mathrm{mol}}$ with redshift, based on previous targeted observations of star-forming galaxies. A fixed conversion factor $\alpha_{\mathrm{CO}}=3.6\left(\mathrm{~K} \mathrm{~km} \mathrm{~s}^{-1} \mathrm{pc}^{2}\right)^{-1}$ has been assumed for the ASPECS $\mathrm{CO}$ sources. The comparison sample uses a metallicity-based prescription for this parameter. Typical values will range between $\alpha_{\mathrm{CO}}=2-5 M_{\odot}\left(\mathrm{K} \mathrm{km} \mathrm{s}^{-1} \mathrm{pc}^{2}\right)^{-1}$ for the ASPECS CO sources.

that of PHIBSS1/2 sources, with a significant fraction of sources having large gas depletion timescales and SSFR below $1 \mathrm{Gyr}^{-1}$, the ASPECS CO galaxies fall well within the lines of constant gas ratio $\left(M_{\mathrm{mol}} / M_{\text {stars }}\right)$ at 0.1 and 10 and overall appear to follow the standard relationship between these quantities. This is more clearly seen in the right panel, which shows the sSFR normalized by the expected sSFR value of the MS (i.e., the offset from the MS), using the MS prescription by Schreiber et al. (2015). Here, the ASPECS CO-selected galaxies follow the standard linear trend, supporting a direct connection between the distance from the MS and the gas depletion timescale (or inversely the star formation efficiency). The large span of properties of ASPECS galaxies suggests that a wider parameter space exists beyond that explored by targeted gas/dust observations of preselected galaxies.

Figure 10 shows the molecular gas depletion timescales and molecular gas ratios of ASPECS CO galaxies as a function of redshift, color-coded by stellar mass, compared to the $z>1$ PHIBSS 1/2 CO sample. The ASPECS CO-selected galaxies do not show a particular trend of $t_{\text {dep }}$ with redshift, and within the uncertainties they seem consistent with the predicted mild evolution of this parameter. As also shown in Figure 8, the ASPECS CO galaxies display a significant span in $t_{\text {dep }}$ compared to the PHIBSS1/2 sample. A stronger evolution is seen in terms of $M_{\text {mol }} / M_{\text {stars }}$. If we focus only on the more massive galaxies, depicted as green and red points, there is an obvious increase in the average value of the molecular gas ratio from $M_{\text {mol }} / M_{\text {stars }} \sim 0.3$ at $z=1$ to $\sim 2$ at $z=2.5$. The ASPECS CO-selected sample supports the strong evolution in molecular gas ratio (or fraction) expected from previous targeted observations and models.

\subsection{Molecular Gas Budget}

Inspection of Figure 9 and the color-coding of the data points, suggests there is a tendency of having more starbursting galaxies with increasing redshift (i.e., higher values of sSFR with increasing redshift). Conversely, galaxies tend to be more passive at lower redshifts. This effect is expected by standard scaling relations and has been seen by previous targeted $\mathrm{CO}$ 


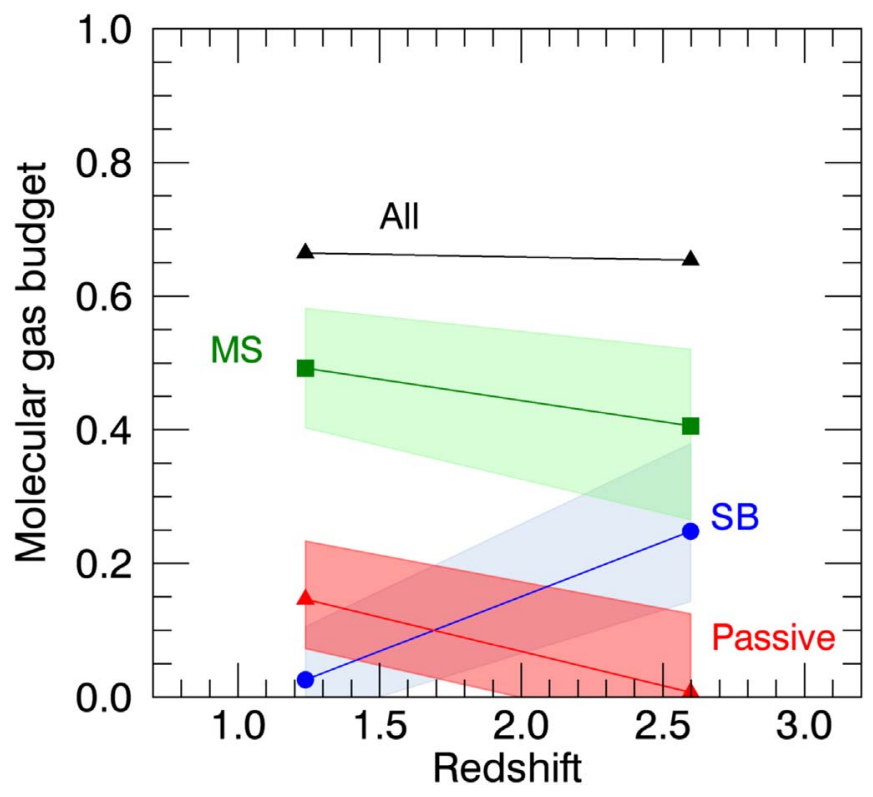

Figure 11. Contribution to the total molecular gas budget from galaxies above (starburst), in, or below (passive) the MS as a function of redshift inferred from the ASPECS survey. The blue, green, and red data points and lines represent galaxies above, in, and below the MS, respectively. The black curve shows the contribution of all the $\mathrm{CO}$-selected galaxies considered here to the total molecular gas at each redshift. Each data point is computed from the sum of molecular gas masses of all galaxies in that redshift bin and galaxy type. The redshift measurement of each point is computed as the average redshift from all galaxies in that bin. The shaded region corresponds to the uncertainties of each measurement.

surveys (e.g., Tacconi et al. 2013, 2018). The clean CO-based selection of the ASPECS survey now allows us to investigate how the total budget of molecular gas in galaxies evolves as a function of redshift and distance from the MS (i.e., galaxy type).

We divided the ASPECS sample into three sets: galaxies significantly above the MS, with $\log \left(\delta_{\mathrm{MS}}\right)=\log \left(\mathrm{sSFR} / \mathrm{sSFR}_{\mathrm{MS}}\right)$ above 0.4 ("starburst"); galaxies below the MS, with $\log \left(\delta_{\mathrm{MS}}\right)<-0.4$ ("passive"); and galaxies within the MS, with $-0.4<\log \left(\delta_{\mathrm{MS}}\right)<0.4$ ("MS"). We subdivide these samples into two broad redshift bins: $1.0<z<1.7 ; 2.0<z<3.1$, which essentially trace the redshift coverage of ASPECS for $\mathrm{CO}(2-1)$ and $\mathrm{CO}(3-2)$. Each of these redshift bins contains 10 and 4 sources, respectively (sources $3 \mathrm{~mm} .8$ was excluded due to the ambiguous optical identification and $3 \mathrm{~mm} .13$ due to its redshift outside the defined range). For each redshift bin, we now ask the question of what is the contribution of each galaxy type to the total budget of molecular gas (or what fraction of the total budget they are making up). At each redshift bin, we thus compute this contribution as the sum of all the molecular gas masses from galaxies of this particular type divided by the total molecular gas mass obtained from the recent measurement of cosmic molecular gas density $\left(\rho_{\mathrm{H}_{2}}\right)$ using ASPECS data (Decarli et al. 2019).

The result of this exercise is shown in Figure 11. Here, the different colors represent the galaxy types, and the shaded regions corresponds to the associated uncertainties in these measurements. The values of redshifts used in the horizontal axes correspond to the average redshift among all galaxies in that redshift bin. These uncertainties in the vertical axes are computed as the sum in quadrature of the individual molecular gas mass values, added in quadrature to the statistical uncertainty, which follows binomial distribution, scaled to the total molecular gas in that redshift bin.

The fact that we do not reach full completeness when adding up all CO-selected ASPECS sources is due to the fact that the total molecular gas density also accounts for fainter galaxies that are not part of our sample.

While the analysis is still limited by the admittedly low number of sources (and thus large statistical uncertainties), there appears to be a difference in the trends followed by the different galaxy types. MS galaxies seem to have a dominant contribution to the molecular gas mass budget, which tends to slightly decrease at high redshifts. This decrease, however, is likely driven by the drop in the total contribution from our bright ASPECS galaxies (black curve). Starburst galaxies are consistent with mild evolution, with a contribution increasing from $\sim 5 \%$ at $z \sim 1.2$ to $\sim 20 \%$ at higher redshift (yet still consistent with no evolution at $1 \sigma$ ). Passive galaxies appear to have a decreasing contribution with increasing redshift, falling from $15 \%$ at $z \sim 1.2$ to $0 \%$ at $z \sim 2.6$.

Current IR surveys indicate that starburst galaxies have a relatively constant, yet minor, contribution to the cosmic SFR density as a function of redshift, of $~ 8 \%-14 \%$ (Sargent et al. 2012; Schreiber et al. 2015), whereas MS galaxies would have a dominant contribution out to $z=2$. This is consistent with the results presented here in terms of the contribution of starburst and MS galaxies to the molecular gas budget with redshift, and this consistency is expected if the molecular gas content is directly linked to the star formation activity in these kinds of galaxies, except only if there is substantial change in efficiencies by a particular galaxy type. However, the decreasing contribution with increasing redshift found for passive galaxies seems to be in contradiction with recent findings by Gobat et al. (2018) that quiescent early-type galaxies at $z=1.8$ have two orders of magnitude more dust than early-type galaxies at $z \sim 0$. As argued by these authors, this result implies the presence of leftover molecular gas in these $z \sim 1.8$ quiescent galaxies, which is consumed in a low-efficient fashion.

This discrepancy can be understood as follows. Starburst galaxies, typically more abundant at $z>1$, would rapidly exhaust most of their molecular gas reservoirs and typically evolve into passive galaxies. The latter would be more numerous at lower redshifts $(z \sim 1)$, and might still retain some of the leftover molecular gas from the previous starburst episode(s) (as pointed out by Gobat et al. 2018). Hence, while passive galaxies might have on average significantly more molecular gas at higher redshifts $(z \sim 2-3)$, they still represent a very minor fraction of the cosmic molecular gas density or molecular gas budget compared to MS or starburst galaxies. At lower redshifts $(z \sim 1)$ passive galaxies would have already consumed part of their molecular gas reservoirs; however, because they are more numerous, they would contribute an increasing fraction to the cosmic molecular gas density. These "below MS" galaxies would thus not only be more prone to be detected by surveys like ASPECS. Perhaps most importantly, this reflects the possibly important, yet overlooked, role of these kinds of galaxies in the formation of stars in the universe.

\section{Conclusions}

We have presented an analysis of the molecular gas properties of a sample of $16 \mathrm{CO}$ line selected galaxies in the 
ALMA Spectroscopic Survey in the Hubble UDF, plus two additional CO line emitters identified through optical MUSE spectroscopy.

The ASPECS CO-selected galaxies follow a tight relationship in the CO luminosity versus FWHM plane, suggestive of disk-like morphologies in most cases. We find that the ASPECS CO galaxies span a range in properties compared to previous preselected galaxies with $\mathrm{CO} /$ dust follow-up observations. Our galaxies are found to lie at $z \sim 1-4$, with stellar masses in the range $(0.03-4) \times 10^{11} M_{\odot}$, SFRs in the range $(0-300) M_{\odot} \mathrm{yr}^{-1}$ and gas masses in the range $5 \times 10^{9} M_{\odot}$ to $1.1 \times 10^{11} M_{\odot}$. The wide range of properties shown by the ASPECS CO galaxies expand the range covered by PHIBSS1/ 2 in $\mathrm{CO}$ at $z>1$, with two galaxies falling significantly below the MS $(\sim 15 \%)$ and the other three sources $(\sim 20 \%)$ above the MS at their respective redshifts.

The ASPECS CO galaxies are found to tightly follow the SFR $-M_{\text {mol }}$ relation, with a typical molecular gas depletion timescale of $1 \mathrm{Gyr}$, similar to $z>1$ PHIBSS $1 / 2 \mathrm{CO}$ galaxies, yet spanning a range from 0.1 to $10 \mathrm{Gyr}$. Similarly, the ASPECS sources are found to span a wide range in molecular gas ratios ranging from $M_{\mathrm{mol}} / M_{\mathrm{stars}}=0.2$ to 6.0. Despite the wide range of properties, the ASPECS CO-selected sources follow remarkably the standard scaling relation trends of $t_{\mathrm{dep}}$ and $\mu_{\mathrm{mol}}$ with sSFR and redshift.

Finally, we take advantage of the nature of the ASPECS survey to measure the contribution of the molecular gas budget as a function of redshift from galaxies above, in, and below the MS. We find a dominant role from MS galaxies. Starburst galaxies appear to have a relatively flat contribution of $\sim 10 \%$ at $z=1$ and $z=2$. Conversely, passive galaxies appear to have a relevant contribution to the molecular gas budget at $z<1$, yet almost none at $z>1$. We argue this could be due to starbursts evolving into passive galaxies at $z \sim 1$, and thus an increasing number of passive galaxies with leftover molecular gas.

We thank Linda Tacconi for making the PHIBSS $1 / 2$ compilation available. We also thank the anonymous referee for helpful and constructive comments. "Este trabajo contó con el apoyo de CONICYT + Programa de Astronomía+ Fondo CHINA-CONICYT." J.G.L. acknowledges partial support from ALMA-CONICYT project 31160033. F.E.B. acknowledges support from CONICYT grant Basal AFB-170002 (F.E.B.), and the Ministry of Economy, Development, and Tourism's Millennium Science Initiative through grant IC120009, awarded to The Millennium Institute of Astrophysics, MAS (F.E.B.). T.D.S. acknowledges support from ALMA-CONICYT project 31130005 and FONDECYT project 1151239. J.H. acknowledges support of the VIDI research programme with project number 639.042.611, which is (partly) financed by the Netherlands Organization for Scientific Research (NWO) D.R. acknowledges support from the National Science Foundation under grant number AST1614213. I.R.S. acknowledges support from the ERC Advanced Grant DUSTYGAL (321334) and STFC (ST/ P000541/1). This paper makes use of the following ALMA data: 2016.1.00324.L. ALMA is a partnership of ESO (representing its member states), NSF (USA) and NINS (Japan), together with NRC (Canada), NSC and ASIAA (Taiwan), and KASI (Republic of Korea), in cooperation with the Republic of Chile. The Joint ALMA Observatory is operated by ESO, AUI/NRAO, and NAOJ.

\section{Appendix A Search for [C I] Line Emission}

The line identification for one of the ASPECS CO detections, $3 \mathrm{~mm} .13$, was found to be consistent with $\mathrm{CO}(4-3)$ at a redshift of 3.601, based on the comparison with the photometric redshift estimate (Boogaard et al. 2019). At this redshift, the $3 \mathrm{~mm}$ band also covers the [C I] 1-0 emission line. We extracted a spectral profile around the expected frequency of this line; however, no line detection is found down to an rms of $0.26 \mathrm{mJy}_{\text {beam }}^{-1}$ per $21 \mathrm{~km} \mathrm{~s}^{-1}$ channel or $0.09 \mathrm{mJy}^{-1}$ beam ${ }^{-1}$ per $200 \mathrm{~km} \mathrm{~s}^{-1}$ channel. This places a limit on the line luminosity, assuming the [C I] line would have the same width as $\mathrm{CO}(4-3)$, of $L_{[\mathrm{C}]]}^{\prime}=2.7 \times$ $10^{9} \mathrm{~K} \mathrm{~km} \mathrm{~s}^{-1} \mathrm{pc}^{2}(3 \sigma)$. Following Bothwell et al. (2017), we compute an upper limit to the molecular gas mass from this [C I] line measurement (see also Papadopoulos et al. 2004; Wagg et al. 2006) using:

$$
M\left(\mathrm{H}_{2}\right)^{\mathrm{CI}}=1375.8\left(\frac{D_{\mathrm{L}}^{2}}{(1+z)}\right)\left(\frac{X_{[\mathrm{C} \mathrm{I}]}}{10^{-5}}\right)^{-1}\left(\frac{A_{10}}{10^{-7}}\right)^{-1} Q_{10}^{-1} F_{[\mathrm{C} \mathrm{I}]}
$$

where $D_{\mathrm{L}}$ is the luminosity distance in $\mathrm{Mpc}, X_{[\mathrm{C} \mathrm{I}]}$ is the $[\mathrm{C} \mathrm{I}] / \mathrm{H}_{2}$ abundance ratio, which we assume to be $3 \times 10^{5}$, and $A_{10}$ is the Einstein A coefficient equal to $7.93 \times 10^{-8} \mathrm{~s}^{-1}$. $Q_{10}$ is the excitation factor, which we set at 0.6 and $F_{[\mathrm{C} \text { I] }}$ is the [C I] line intensity in units of $\mathrm{Jy} \mathrm{km} \mathrm{s}^{-1}$. Thus, we find a $3 \sigma$ limit for the [C I]-based molecular gas mass $M\left(\mathrm{H}_{2}\right)^{\mathrm{CI}}<1.9 \times$ $10^{10} M_{\odot}$. This limit is consistent with the molecular gas mass estimate derived from $\mathrm{CO}$ of $1.3 \times 10^{10} M_{\odot}$. Note that this estimate extrapolates the $\mathrm{CO}(4-3)$ line emission down to $\mathrm{CO}(1-0)$ using a template obtained for massive BzK galaxies at $z=1.5$. If the CO SLED is steeper, with $\mathrm{CO}(4-3)$ and $\mathrm{CO}(1-0)$ closer to thermal equilibrium, the $\mathrm{CO}$-derived gas mass would be in better agreement.

\section{Appendix B \\ Flux Measurements in Tapered Cubes}

We explored the possibility that we could be missing some flux due to sources being extended spatially. For this, we created a new version of the ASPECS band-3 data cube, tapered to an angular resolution of $\sim 3^{\prime \prime}$, which should contain all of the extended CO line emission. We collapsed this cube, created the moment -0 images, and computed the integrated fluxes as the value of the peak pixels in these images. Figure 12 shows the comparison of $\mathrm{CO}$ fluxes and line widths between these two estimates. The flux estimates for almost all sources are in excellent agreement within the uncertainties.

In the case of source $3 \mathrm{~mm} .16$, the measurement in the tapered cube not only doubles the flux in the original one, but also yields a much larger line FWHM. This suggests significant extended low surface brightness emission, undetected in the original cube. Manual inspection of the cube, however, shows that the extra emission can be attributed to noise at large velocities $\left(>200 \mathrm{~km} \mathrm{~s}^{-1}\right)$. All the other sources, however, have an excellent agreement between their measured FWHM. We thus use the original flux estimates throughout this paper. 

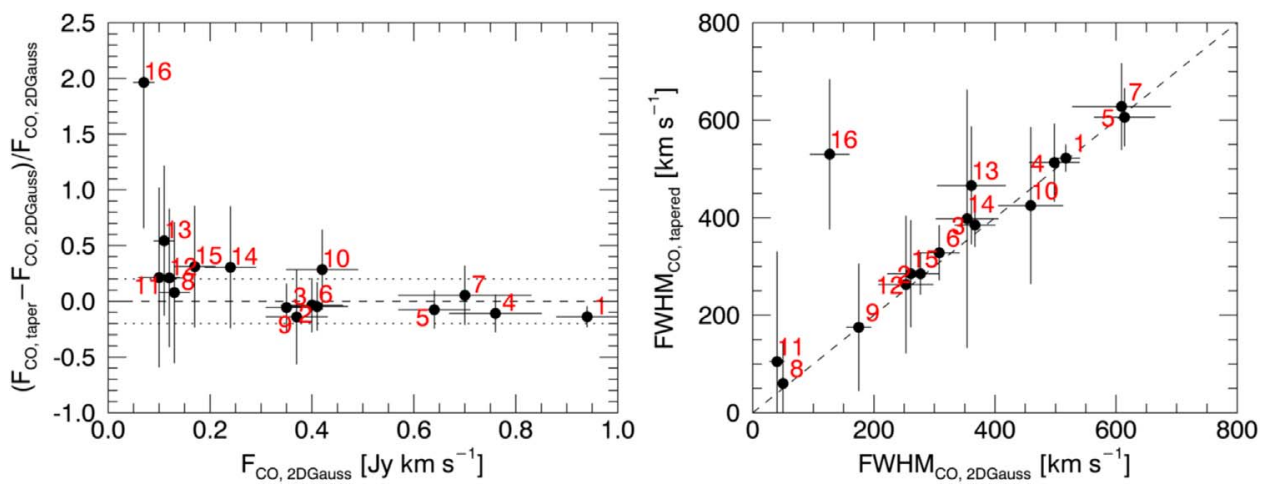

Figure 12. Comparison of the $\mathrm{CO}$ flux measurements and line widths obtained from two-dimensional Gaussian fitting in the original resolution moment- 0 maps vs. the ones obtained from the measurements in the $3^{\prime \prime}$ tapered cubes. Dotted lines in the top panel indicate lines of $20 \%$ difference between these estimates. The dashed lines indicate the location of identical estimates by both methods.

Table 3

Sizes of the ASPECS CO Galaxies

\begin{tabular}{lcccccc}
\hline \hline $\begin{array}{l}\text { ID } \\
3 \mathrm{~mm} .\end{array}$ & $z_{\mathrm{CO}}$ & $r_{1 / 2, \mathrm{CO}}$ & $\begin{array}{c}e_{\mathrm{CO}} \\
(\mathrm{kpc})\end{array}$ & $\begin{array}{c}r_{1 / 2, \mathrm{opt}} \\
(4)\end{array}$ & $\begin{array}{c}n_{\mathrm{opt}} \\
(\mathrm{kpc})\end{array}$ & $e_{\mathrm{opt}}$ \\
\hline 1 & $(2)$ & $(3)$ & $(5)$ & $(6)$ & \\
\hline 2 & 2.543 & $4.3 \pm 0.4$ & $3.7 \pm 3.4$ & 1.7 & 0.8 & 0.8 \\
3 & 1.317 & $\ldots$ & $\ldots$ & 4.0 & 2.2 & 0.6 \\
4 & 2.453 & $3.9 \pm 0.5$ & $2.2 \pm 0.7$ & 5.1 & 0.2 & 0.3 \\
5 & 1.414 & $5.0 \pm 0.7$ & $3.7 \pm 2.2$ & 7.4 & 0.5 & 0.2 \\
6 & 1.550 & $4.2 \pm 0.7$ & $2.4 \pm 1.1$ & 8.3 & 3.0 & 0.4 \\
7 & 1.095 & $4.5 \pm 1.1$ & $1.2 \pm 0.6$ & 5.4 & 1.1 & 0.8 \\
$8^{\mathrm{b}}$ & 2.697 & $\ldots$ & $\ldots$ & 4.8 & 0.9 & 0.5 \\
9 & 1.382 & $5.3 \pm 1.6$ & $2.1 \pm 1.4$ & $\ldots$ & $\ldots$ & $\ldots$ \\
10 & 2.698 & $\ldots$ & $\ldots$ & 0.6 & 7.2 & 0.7 \\
11 & 1.037 & $3.6 \pm 1.1$ & $2.2 \pm 1.8$ & 2.5 & 0.9 & 0.5 \\
$12^{\mathrm{b}}$ & 1.096 & $3.6 \pm 1.3$ & $4.1 \pm 3.1$ & 1.8 & 0.2 & 0.6 \\
13 & 2.574 & $\ldots$ & $\ldots$ & $\ldots$ & $\ldots$ & $\ldots$ \\
$14^{X}$ & 3.601 & $4.0 \pm 1.2$ & $1.8 \pm 1.5$ & 0.9 & 2.1 & 0.4 \\
15 & 1.098 & $\ldots$ & $\ldots$ & $\ldots$ & $\ldots$ & $\ldots$ \\
16 & 1.096 & $\ldots$ & $\ldots$ & 6.0 & 0.4 & 0.4 \\
\hline
\end{tabular}

Notes.

${ }^{\text {a }}$ For sources that were unresolved in $\mathrm{CO}$ emission, no sizes are provided.

${ }^{\mathrm{b}}$ Sources $3 \mathrm{~mm} .8$ and $3 \mathrm{~mm} .12$ do not have reliable optical counterparts and thus their optical sizes are not listed. ${ }^{X}$ Source $3 \mathrm{~mm} .14$ does not have a reliable optical morphology estimate in the catalog of van der Wel et al. (2012). Columns: (1) Source ID. (2) CO redshift. (2) Half-light radius of the CO emission, assuming a Gaussian distribution (Sérsic index of 0.5). (3) Ellipticity of the $\mathrm{CO}$ distribution. (4) Half-light radius of the optical emission, using a Sérsic profile with index $n_{\text {opt }}$ (van der Wel et al. 2012). (5) Sérsic index of the optical emission. (6) Ellipticity of the Sérsic profile.

\section{Appendix C CO and Optical Sizes}

We used the CO moment- 0 maps at original resolution (no tapering) to measure $\mathrm{CO}$ emitting sizes of the ASPECS sources. Here, we only focus on the 16 brighter CO-selected sources. We used the CASA task imfit to fit two-dimensional Gaussian profiles to these images centered at the $\mathrm{CO}$ source positions. Due to the limited angular resolution and sensitivity of our observations, we did not attempt to fit more complicated profiles, which require more free parameters (i.e., Sérsic profile). From this, we extracted the deconvolved semimajor and semiminor axes of the fitted Gaussian profile $\left(B_{\mathrm{maj}}, B_{\mathrm{min}}\right)$,

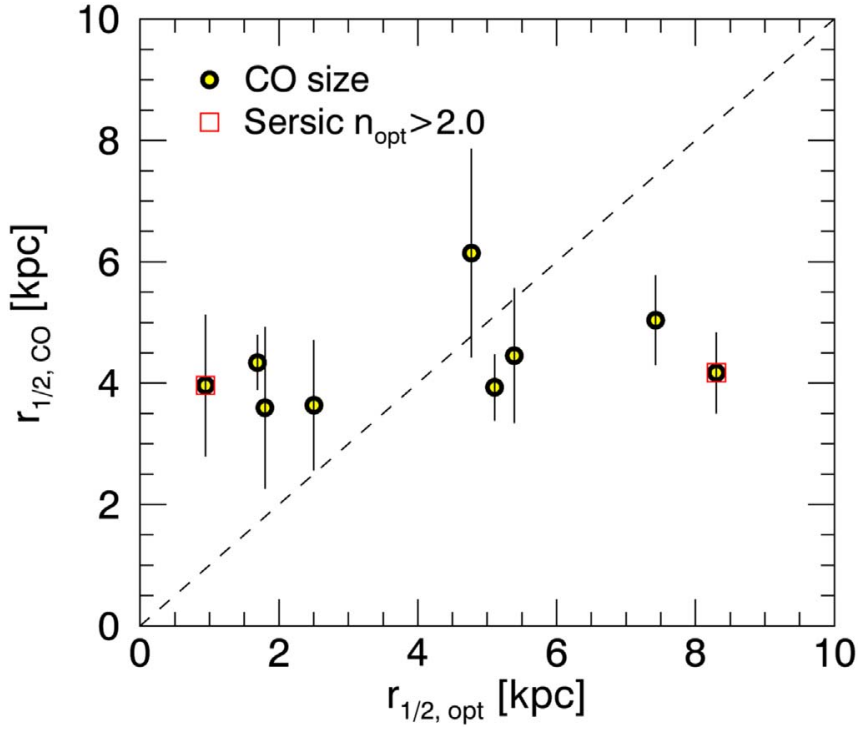

Figure 13. Comparison between the rest-frame optical sizes derived by van der Wel et al. (2012) and the CO sizes measured from the ASPECS data. Only resolved sources in $\mathrm{CO}$ emission are considered. Red squares highlight sources for which the optical morphology indicates large Sercic indexes, which would indicate highly concentrated optical emission and thus might not be directly comparable to the $\mathrm{CO}$ estimates.

and computed the half-light radius $r_{1 / 2}$ by averaging these two (weighted by uncertainties). We computed the ellipticity of the profile as $e=B_{\text {maj }} / B_{\text {min }}$. We consider that the source is resolved in $\mathrm{CO}$ emission if either $B_{\text {maj }}$ or $B_{\text {min }}$ are measured at a significance above 3 . The derived parameters are listed in Table 3

In addition to the $\mathrm{CO}$ sizes, we use the structural parameters derived by van der Wel et al. (2012) from the HST near-IR images of the CANDELS field. We remove sources $3 \mathrm{~mm} .8$ and $3 \mathrm{~mm} .12$ since their optical counterparts are contaminated by foreground structures. The parameters are listed in Table 3 . van der Wel et al. (2012) use Sérsic profiles to fit these images, given the high resolution and signal of the rest-frame optical sources. Note that a two-dimensional Gaussian profile is equivalent to a Sérsic profile with index $n=0.5$. In some cases, the fitted profiles show Sérsic index $n$ values above 2, indicative of a highly concentrated central source (for example, a bright central bulge, or a galactic nuclei). Thus, in these cases 

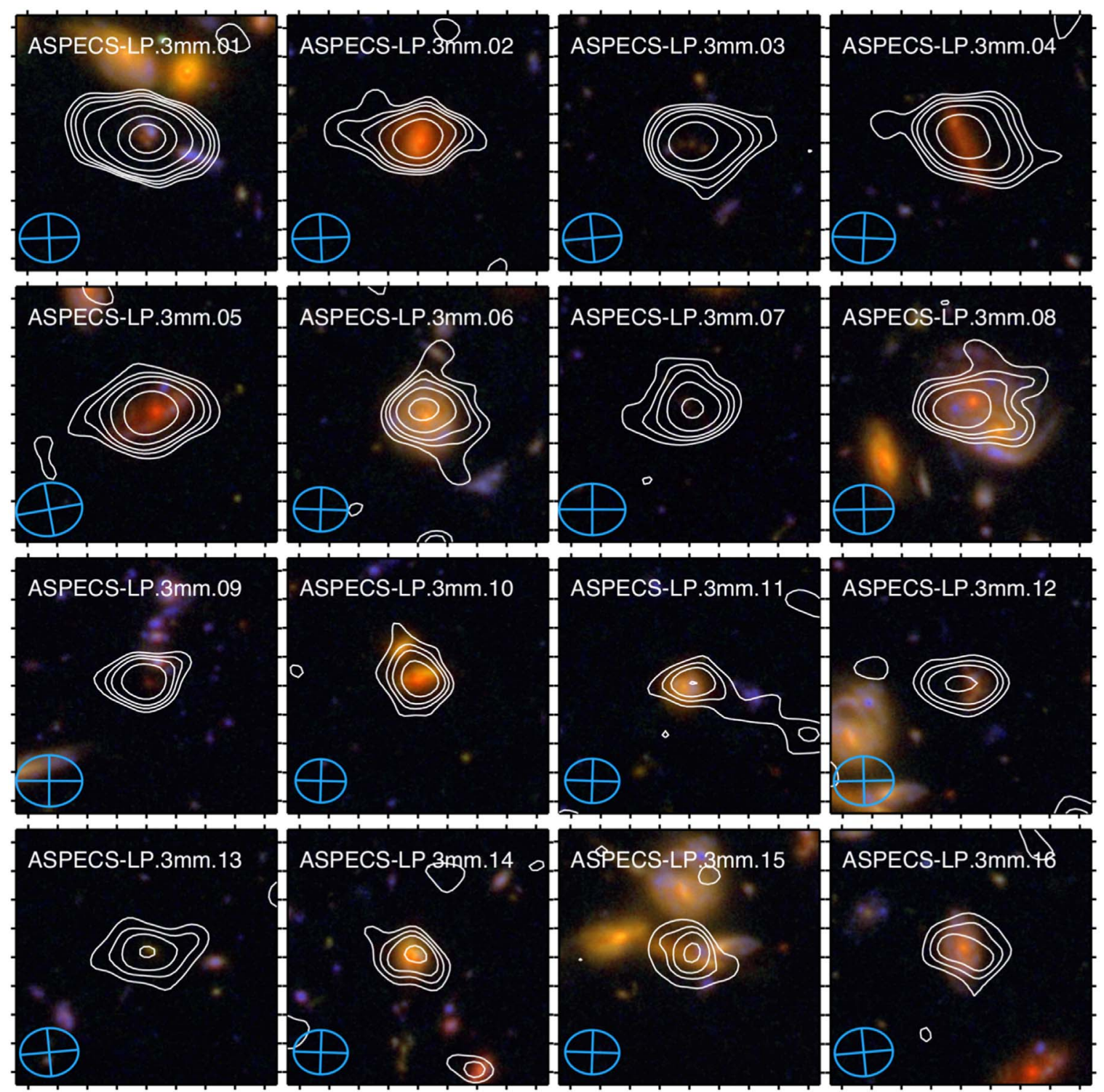

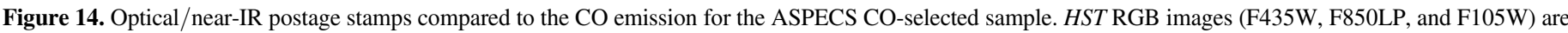

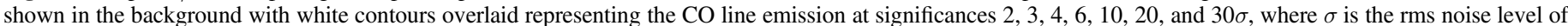
each $\mathrm{CO}$ moment-0 image.

the derived values of the half-light radius, $r_{1 / 2}$, in the $H S T$ images might not be necessarily comparable to the values derived for CO.

Figure 13 compares the $\mathrm{CO}$ and optical sizes $\left(r_{1 / 2}\right)$ derived in this way. Figure 14 shows a visual comparison of the optical/ near-IR with the $\mathrm{CO}$ line emission morphologies. We find no clear correlation between the $\mathrm{CO}$ and the optical sizes. The $\mathrm{CO}$ sizes seem to stay relatively constant around $\sim 4-6 \mathrm{kpc}$, whereas the optical sizes span a significant range from $\sim 1.0$ to $8.5 \mathrm{kpc}$. We note that even in cases where the $\mathrm{CO}$ emission is significantly resolved, as in sources $3 \mathrm{~mm} .1,3 \mathrm{~mm} .3$, or $3 \mathrm{~mm} .5$, the optical sizes show evident differences compared to the $\mathrm{CO}$ sizes. This suggests that (at least for these sources) the differences in size between $\mathrm{CO}$ and optical are physical, and not necessarily driven by the angular resolution and sensitivity limits of our data. This also may suggest that the $\mathrm{CO}$ sizes are relatively homogeneous in our sample. However, this result is limited by the fact that about half of our sample is currently unresolved.

\section{Appendix D \\ CO Kinematics}

Since some of our galaxies were resolved in CO line emission, we computed CO moment-1 maps or velocity fields (see Figure 15). In some cases, we clearly see velocity gradients suggestive of ordered gas rotation $(3 \mathrm{~mm} .4,3 \mathrm{~mm} .5$, $3 \mathrm{~mm} .6$, and $3 \mathrm{~mm} .7$ ). In the particular case of $3 \mathrm{~mm} .7$, the $\mathrm{CO}$ emission is marginally resolved in one axis only, but the velocity field shows the structure clearly. Other cases with hints of velocity gradients are limited by the significance and resolution. Conversely, other cases where the emission is significantly resolved, such as in $3 \mathrm{~mm} .1$, do not show evidence of rotation and suggest a dispersion dominated object.

We take advantage of the software ${ }^{3 D}$ Barolo (Di Teodoro \& Fraternali 2015) to perform a tilted-ring modeling of the gas velocity field. Because of the coarse resolution of our data, we fix the ring inclination and position angle based on the Sérsic fits performed on all the sources in the field by van der Wel et al. (2012), 

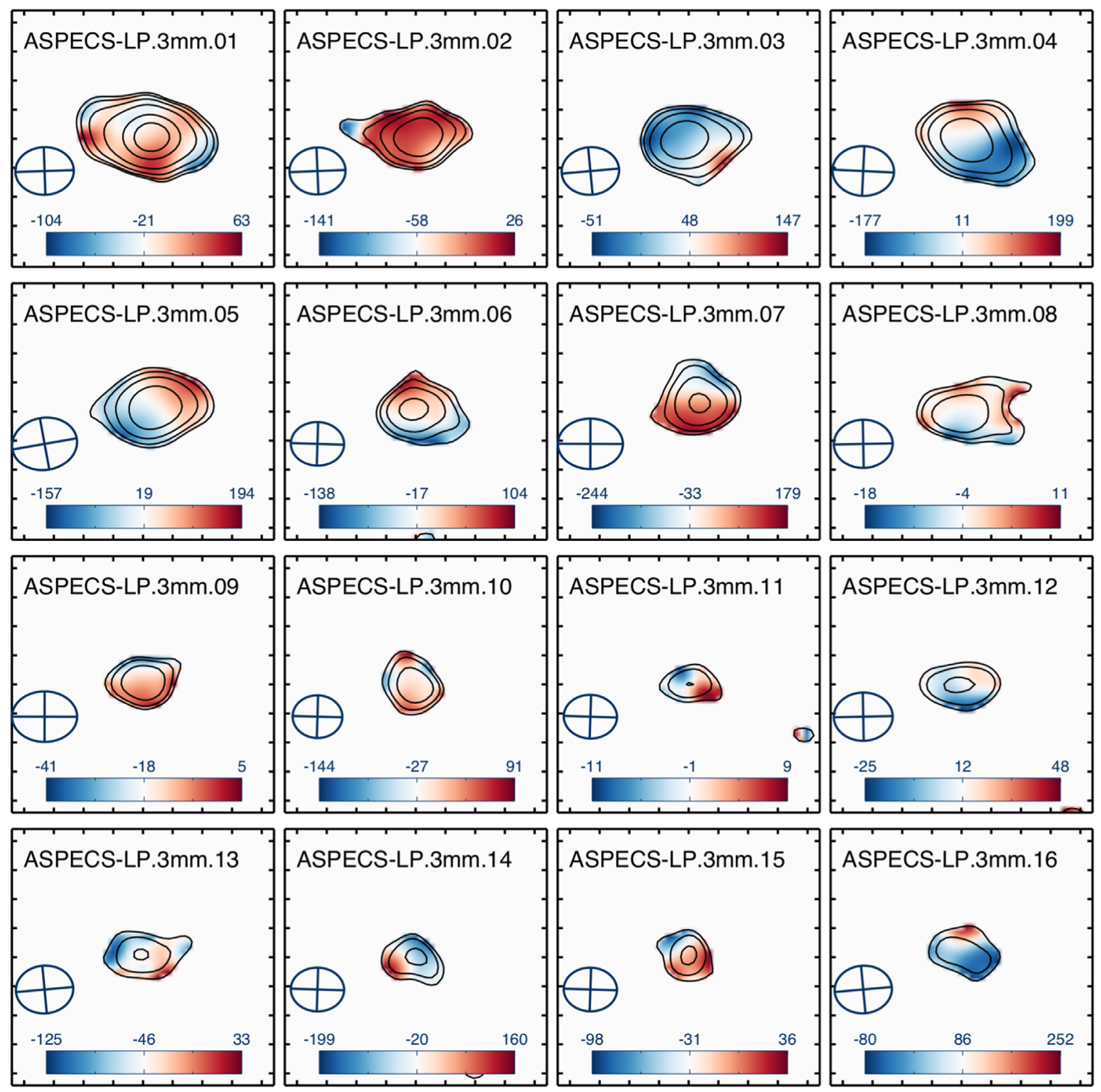

Figure 15. Postage stamps of the CO moment-1 (velocity fields) toward the ASPECS CO sources. The background image represents the velocity field, with bluer and redder colors representing the approaching and receding CO components. The black contours show the moment- 0 map, shown at levels $3,6,10,15,20$, and $30 \sigma$, where $\sigma$ is the rms measured in this map. The color bar presented in the bottom of each panel shows the velocity scale in each case with respect to the CO central velocity, in units of kilometers per second. The blue ellipse to the left side of each panel represents the beam size at the observed frequency.

so that only the centroid of the line emission, the gas velocity, and velocity dispersion are free in the fit.

The ASPECS LP $3 \mathrm{~mm}$ data were obtained in a relatively compact array configuration, thus the majority of the sources are only marginally resolved, and a proper dynamical analysis is not feasible. Only three sources show a significant velocity gradient in the $\mathrm{CO}$ emission, which allows us to put loose constraints on the dynamical mass. The dynamical mass is derived as $M_{\mathrm{dyn}}=R v_{\mathrm{rot}}^{2} G^{-1}$. At $R=R_{\mathrm{opt}}$, the dynamical masses inferred for the ASPECS sources $3 \mathrm{~mm} .4,3 \mathrm{~mm} .5$, and $3 \mathrm{~mm} .7$ are $(8.1 \pm 2.4) \times 10^{10} M_{\odot},(2.7 \pm 0.8) \times 10^{11} M_{\odot}$, and $(1.4 \pm$ $0.5) \times 10^{11} M_{\odot}$, respectively.

\section{ORCID iDs}

Manuel Aravena (1) https://orcid.org/0000-0002-6290-3198 Roberto Decarli (i) https://orcid.org/0000-0002-2662-8803
Jorge Gónzalez-López (i) https://orcid.org/0000-00033926-1411

Leindert Boogaard (1) https://orcid.org/0000-0002-3952-8588 Fabian Walter (1) https://orcid.org/0000-0003-4793-7880 Chris Carilli (1) https://orcid.org/0000-0001-6647-3861 Gergö Popping (1) https://orcid.org/0000-0003-1151-4659 Axel Weiss (1) https://orcid.org/0000-0003-4678-3939 Roberto J. Assef (10) https://orcid.org/0000-0002-9508-3667 Franz Erik Bauer (10) https://orcid.org/0000-0002-8686-8737 Frank Bertoldi 낭 https://orcid.org/0000-0002-1707-1775 Richard Bouwens (1) https://orcid.org/0000-0002-4989-2471 Thierry Contini (1) https://orcid.org/0000-0003-0275-938X Paulo C. Cortes (1) https://orcid.org/0000-0002-3583-780X Emanuele Daddi (1) https://orcid.org/0000-0002-3331-9590 Jacqueline Hodge (i) https://orcid.org/0000-0001-6586-8845 Rob Ivison (10) https://orcid.org/0000-0001-5118-1313 Benjamin Magnelli (i) https://orcid.org/0000-0002-6777-6490 
Pascal Oesch (1) https://orcid.org/0000-0001-5851-6649 Dominik Riechers (i) https://orcid.org/0000-0001-9585-1462 Ian Smail @ https://orcid.org/0000-0003-3037-257X A. M. Swinbank (1) https://orcid.org/0000-0003-1192-5837 Bade Uzgil (1) https://orcid.org/0000-0001-8526-3464

\section{References}

Aravena, M., Decarli, R., Walter, F., et al. 2016b, ApJ, 833, 68 Aravena, M., Decarli, R., Walter, F., et al. 2016c, ApJ, 833, 71 Aravena, M., Spilker, J. S., Bethermin, M., et al. 2016a, MNRAS, 457, 4406 Bacon, R., Conseil, S., Mary, D., et al. 2017, A\&A, 608, A1 Barro, G., Kriek, M., Pérez-González, P. G., et al. 2016, ApJL, 827, L32 Bauermeister, A., Blitz, L., Bolatto, A., et al. 2013, ApJ, 768, 132 Berta, S., Lutz, D., Genzel, R., Förster-Schreiber, N. M., \& Tacconi, L. J. 2016, A\&A, 587, A73

Béthermin, M., Daddi, E., Magdis, G., et al. 2015, A\&A, 573, A113 Bigiel, F., Leroy, A., Walter, F., et al. 2008, AJ, 136, 2846

Bolatto, A. D., Wolfire, M., \& Leroy, A. K. 2013, ARA\&A, 51, 207

Boogaard, L. A., Van der Werf, P., \& Bouwens, R. e. a. 2019, ApJ, 882, 140 Boselli, A., Lequeux, J., \& Gavazzi, G. 2002, Ap\&SS, 281, 127 Bothwell, M. S., Aguirre, J. E., Aravena, M., et al. 2017, MNRAS, 466, 2825 Bothwell, M. S., Smail, I., Chapman, S. C., et al. 2013, MNRAS, 429, 3047 Bouwens, R. J., Aravena, M., Decarli, R., et al. 2016, ApJ, 833, 72 Bouwens, R. J., Bradley, L., Zitrin, A., et al. 2014, ApJ, 795, 126 Brinchmann, J., Charlot, S., White, S. D. M., et al. 2004, MNRAS, 351, 1151 Carilli, C. L., \& Blain, A. W. 2002, ApJ, 569, 605

Carilli, C. L., Chluba, J., Decarli, R., et al. 2016, ApJ, 833, 73 Carilli, C. L., Hodge, J., Walter, F., et al. 2011, ApJL, 739, L33 Carilli, C. L., \& Walter, F. 2013, ARA\&A, 51, 105

Coe, D., Benítez, N., Sánchez, S. F., et al. 2006, AJ, 132, 926 Combes, F., García-Burillo, S., Braine, J., et al. 2011, A\&A, 528, A124

Combes, F., García-Burillo, S., Braine, J., et al. 2013, A\&A, 550, A41 Conroy, C. 2013, ARA\&A, 51, 393

Coppin, K. E. K., Chapman, S. C., Smail, I., et al. 2010, MNRAS, 407, L103 da Cunha, E., Charlot, S., \& Elbaz, D. 2008, MNRAS, 388, 1595 da Cunha, E., Walter, F., Smail, I. R., et al. 2015, ApJ, 806, 110 Daddi, E., Bournaud, F., Walter, F., et al. 2010b, ApJ, 713, 686 Daddi, E., Dannerbauer, H., Liu, D., et al. 2015, A\&A, 577, A46 Daddi, E., Dickinson, M., Morrison, G., et al. 2007, ApJ, 670, 156 Daddi, E., Elbaz, D., Walter, F., et al. 2010a, ApJL, 714, L118 Davé, R., Finlator, K., \& Oppenheimer, B. D. 2012, MNRAS, 421, 98 De Breuck, C., Williams, R. J., Swinbank, M., et al. 2014, A\&A, 565, A59 Decarli, R., Walter, F., Aravena, M., et al. 2016a, ApJ, 833, 69 Decarli, R., Walter, F., Aravena, M., et al. 2016b, ApJ, 833, 70 Decarli, R., Walter, F., Carilli, C., et al. 2014, ApJL, 782, L17 Decarli, R., Walter, F., Gonzalez-Lopez, J., et al. 2019, ApJ, 882, 138 Dekel, A., Birnboim, Y., Engel, G., et al. 2009, Natur, 457, 451 Di Teodoro, E. M., \& Fraternali, F. 2015, MNRAS, 451, 3021 Downes, D., \& Solomon, P. M. 1998, ApJ, 507, 615

Dunlop, J. S., McLure, R. J., Biggs, A. D., et al. 2017, MNRAS, 466, 861 Elbaz, D., Daddi, E., Le Borgne, D., et al. 2007, A\&A, 468, 33 Elbaz, D., Dickinson, M., Hwang, H. S., et al. 2011, A\&A, 533, A119 Erb, D. K., Steidel, C. C., Shapley, A. E., et al. 2006, ApJ, 646, 107 Frayer, D. T., Koda, J., Pope, A., et al. 2008, ApJL, 680, L21 Freundlich, J., Combes, F., Tacconi, L. J., et al. 2019, A\&A, 622, A105 Gao, Y., \& Solomon, P. M. 2004, ApJ, 606, 271

García-Burillo, S., Usero, A., Alonso-Herrero, A., et al. 2012, A\&A, 539, A8 Genzel, R., Tacconi, L. J., Combes, F., et al. 2012, ApJ, 746, 69

Genzel, R., Tacconi, L. J., Gracia-Carpio, J., et al. 2010, MNRAS, 407, 2091 Genzel, R., Tacconi, L. J., Lutz, D., et al. 2015, ApJ, 800, 20

Gobat, R., Daddi, E., Magdis, G., et al. 2018, NatAs, 2, 239

González-López, J., Bauer, F. E., Romero-Cañizales, C., et al. 2017, A\&A, 597, A41

González-López, J., Decarli, R., Ricardo, P., et al. 2019, ApJ, 882, 139 Graciá-Carpio, J. 2009, PhD thesis, Universidad Autónoma de Madrid Graciá-Carpio, J., García-Burillo, S., Planesas, P., Fuente, A., \& Usero, A. 2008, A\&A, 479, 703

Greve, T. R., Bertoldi, F., Smail, I., et al. 2005, MNRAS, 359, 1165

Harris, A. I., Baker, A. J., Frayer, D. T., et al. 2012, ApJ, 752, 152
Hodge, J. A., Carilli, C. L., Walter, F., Daddi, E., \& Riechers, D. 2013, ApJ, 776, 22

Inami, H., Bacon, R., Brinchmann, J., et al. 2017, A\&A, 608, A2 Ivison, R. J., Papadopoulos, P. P., Smail, I., et al. 2011, MNRAS, 412, 1913 Ivison, R. J., Swinbank, A. M., Smail, I., et al. 2013, ApJ, 772, 137 Kartaltepe, J. S., Dickinson, M., Alexander, D. M., et al. 2012, ApJ, 757, 23 Kereš, D., Katz, N., Weinberg, D. H., \& Davé, R. 2005, MNRAS, 363, 2 Leroy, A. K., Bolatto, A., Gordon, K., et al. 2011, ApJ, 737, 12 Leroy, A. K., Walter, F., Sandstrom, K., et al. 2013, AJ, 146, 19 Lilly, S. J., Carollo, C. M., Pipino, A., Renzini, A., \& Peng, Y. 2013, ApJ, 772,119

Magdis, G. E., Daddi, E., Béthermin, M., et al. 2012, ApJ, 760, 6 Magdis, G. E., Rigopoulou, D., Daddi, E., et al. 2017, A\&A, 603, A93 Magnelli, B., Lutz, D., Saintonge, A., et al. 2014, A\&A, 561, A86 Magnelli, B., Saintonge, A., Lutz, D., et al. 2012, A\&A, 548, A22

McLure, R. J., Dunlop, J. S., Bowler, R. A. A., et al. 2013, MNRAS, 432, 2696 Mobasher, B., Dahlen, T., Ferguson, H. C., et al. 2015, ApJ, 808, 101

Momcheva, I. G., Brammer, G. B., van Dokkum, P. G., et al. 2015, ApJS, 219, 29

Morris, A. M., Kocevski, D. D., Trump, J. R., et al. 2015, AJ, 149, 178 Noeske, K. G., Weiner, B. J., Faber, S. M., et al. 2007, ApJL, 660, L43 Omont, A. 2007, RPPh, 70, 1099

Papadopoulos, P. P., Thi, W.-F., \& Viti, S. 2004, MNRAS, 351, 147 Papovich, C., Labbé, I., Glazebrook, K., et al. 2016, NatAs, 1, 0003 Pavesi, R., Sharon, C. E., Riechers, D. A., et al. 2018, ApJ, 864, 49 Peng, C. Y., Ho, L. C., Impey, C. D., \& Rix, H.-W. 2010, AJ, 139, 2097 Popping, G., Somerville, R. S., Somerville, R. S., et al. 2019, ApJ, 882, 137 Rhoads, J. E., Malhotra, S., Pirzkal, N., et al. 2009, ApJ, 697, 942 Riechers, D. A., Carilli, C. L., Capak, P. L., et al. 2014, ApJ, 796, 84 Riechers, D. A., Carilli, C. L., Walter, F., \& Momjian, E. 2010, ApJL, 724, L153 Riechers, D. A., Cooray, A., Omont, A., et al. 2011, ApJL, 733, L12 Riechers, D. A., Pavesi, R., Sharon, C. E., et al. 2019, ApJ, 872, 7 Rodighiero, G., Cimatti, A., Gruppioni, C., et al. 2010, A\&A, 518, L25 Saintonge, A., Catinella, B., Cortese, L., et al. 2016, MNRAS, 462, 1749 Saintonge, A., Catinella, B., Tacconi, L. J., et al. 2017, ApJS, 233, 22 Saintonge, A., Kauffmann, G., Kramer, C., et al. 2011a, MNRAS, 415, 32 Saintonge, A., Kauffmann, G., Wang, J., et al. 2011b, MNRAS, 415, 61 Saintonge, A., Lutz, D., Genzel, R., et al. 2013, ApJ, 778, 2

Santini, P., Maiolino, R., Magnelli, B., et al. 2014, A\&A, 562, A30 Sargent, M. T., Béthermin, M., Daddi, E., \& Elbaz, D. 2012, ApJL, 747, L31 Sargent, M. T., Daddi, E., Béthermin, M., et al. 2014, ApJ, 793, 19 Schenker, M. A., Robertson, B. E., Ellis, R. S., et al. 2013, ApJ, 768, 196 Schinnerer, E., Groves, B., Sargent, M. T., et al. 2016, ApJ, 833, 112 Schreiber, C., Pannella, M., Elbaz, D., et al. 2015, A\&A, 575, A74 Schruba, A., Leroy, A. K., Walter, F., et al. 2012, AJ, 143, 138 Scoville, N., Lee, N., Vanden Bout, P., et al. 2017, ApJ, 837, 150 Scoville, N., Sheth, K., Aussel, H., et al. 2016, ApJ, 820, 83

Silverman, J. D., Daddi, E., Rodighiero, G., et al. 2015, ApJL, 812, L23

Skelton, R. E., Whitaker, K. E., Momcheva, I. G., et al. 2014, ApJS, 214, 24 Solomon, P. M., Downes, D., Radford, S. J. E., \& Barrett, J. W. 1997, ApJ, 478,144

Solomon, P. M., \& Vanden Bout, P. A. 2005, ARA\&A, 43, 677

Speagle, J. S., Steinhardt, C. L., Capak, P. L., \& Silverman, J. D. 2014, ApJS, 214,15

Tacconi, L. J., Genzel, R., Saintonge, A., et al. 2018, ApJ, 853, 179

Tacconi, L. J., Genzel, R., Neri, R., et al. 2010, Natur, 463, 781

Tacconi, L. J., Genzel, R., Smail, I., et al. 2008, ApJ, 680, 246

Tacconi, L. J., Neri, R., Chapman, S. C., et al. 2006, ApJ, 640, 228

Tacconi, L. J., Neri, R., Genzel, R., et al. 2013, ApJ, 768, 74

Tadaki, K.-i., Genzel, R., Kodama, T., et al. 2017, ApJ, 834, 135

Tadaki, K.-i., Kohno, K., Kodama, T., et al. 2015, ApJL, 811, L3

Thomson, A. P., Ivison, R. J., Smail, I., et al. 2012, MNRAS, 425, 2203 van der Wel, A., Bell, E. F., Häussler, B., et al. 2012, ApJS, 203, 24

Wagg, J., Wilner, D. J., Neri, R., Downes, D., \& Wiklind, T. 2006, ApJ, 651, 46 Walter, F., Decarli, R., Aravena, M., et al. 2016, ApJ, 833, 67 Walter, F., Decarli, R., Sargent, M., et al. 2014, ApJ, 782, 79

Whitaker, K. E., Franx, M., Leja, J., et al. 2014, ApJ, 795, 104

Whitaker, K. E., van Dokkum, P. G., Brammer, G., \& Franx, M. 2012, ApJL, 754, L29

Wiklind, T., Ferguson, H. C., Guo, Y., et al. 2019, ApJ, 878, 83

Wilson, C. D. 1995, ApJL, 448, L97

Xu, C., Pirzkal, N., Malhotra, S., et al. 2007, AJ, 134, 169 